\title{
On the Completeness of Complete Markets
}

Citation for published version (APA):

Herings, P. J. J., \& Rohde, K. I. M. (2008). On the Completeness of Complete Markets. Economic Theory, 37, 171-202. https://doi.org/10.1007/s00199-007-0291-y

Document status and date:

Published: 01/01/2008

DOI:

10.1007/s00199-007-0291-y

Document Version:

Publisher's PDF, also known as Version of record

Document license:

Taverne

Please check the document version of this publication:

- A submitted manuscript is the version of the article upon submission and before peer-review. There can be important differences between the submitted version and the official published version of record.

People interested in the research are advised to contact the author for the final version of the publication, or visit the DOI to the publisher's website.

- The final author version and the galley proof are versions of the publication after peer review.

- The final published version features the final layout of the paper including the volume, issue and page numbers.

Link to publication

\footnotetext{
General rights rights.

- You may freely distribute the URL identifying the publication in the public portal. please follow below link for the End User Agreement:

www.umlib.nl/taverne-license

Take down policy

If you believe that this document breaches copyright please contact us at:

repository@maastrichtuniversity.nl

providing details and we will investigate your claim.
}

Copyright and moral rights for the publications made accessible in the public portal are retained by the authors and/or other copyright owners and it is a condition of accessing publications that users recognise and abide by the legal requirements associated with these

- Users may download and print one copy of any publication from the public portal for the purpose of private study or research.

- You may not further distribute the material or use it for any profit-making activity or commercial gain

If the publication is distributed under the terms of Article $25 \mathrm{fa}$ of the Dutch Copyright Act, indicated by the "Taverne" license above, 


\title{
On the completeness of complete markets
}

\author{
P. Jean-Jacques Herings • Kirsten I. M. Rohde
}

Received: 10 November 2006 / Accepted: 17 September 2007 / Published online: 16 October 2007

(C) Springer-Verlag 2007

\begin{abstract}
We reconsider the allocational invariance of equilibria to different formulations of market completeness. We identify the so-far neglected assumption of sophisticated behavior as being crucial. First, the Arrow-Debreu setting is considered, where markets do not reopen in the future. Second, sequentially complete markets are analyzed, where goods on the spot markets and all contingent one-period ahead commodities can be traded in every state. Finally, complete markets are analyzed, where all possible contingent commodities can be traded at every state. Preferences may be time-consistent or time-inconsistent. A distinction is made between naïve and sophisticated behavior.
\end{abstract}

Keywords Time-inconsistency · Competitive equilibrium · Market completeness · Allocational equivalence

JEL Classification D51 - D61 - D91

\section{Introduction}

Debreu (1959) discusses how the one-period Arrow-Debreu (1954) model can be used to analyze multi-period settings with uncertainty. The crucial idea is to include

The author would like to thank the Netherlands Organisation for Scientific Research (NWO) for financial support.

\section{P. J.-J. Herings $(\varangle)$}

Department of Economics, Maastricht University, P.O. Box 616, 6200 MD Maastricht, The Netherlands e-mail: P.Herings@algec.unimaas.nl

K. I. M. Rohde

Department of Economics, H13-27, Erasmus University, P.O. Box 1738, 3000 DR Rotterdam,

The Netherlands

e-mail: rohde@few.eur.nl 
the period and the state of the world at the time of delivery in the definition of a commodity, resulting in contingent commodities. Debreu assumes that markets are complete in the sense that all contingent commodities can be traded in the first period and does not allow for markets to reopen after the first period.

As an alternative to the assumption that all contingent commodities can be traded in the initial period, sequentially complete markets have been considered. In a sequentially complete market structure, it is typically assumed that at every state there are complete spot markets for commodities available at that state together with a restricted set of markets for commodities available at future states. It is well-known that, under certain assumptions, equilibria in the Arrow-Debreu model are allocationally equivalent to equilibria in sequentially complete models, see Arrow (1953). Donaldson and Selden (1981) and Haller (1990) provide two discussions of the assumptions needed for this result. Drèze and Herings (2003) show in an example that there might be equilibrium continuations in a sequentially complete markets setting that are not allocationally equivalent to any equilibrium in the Arrow-Debreu setting. A closer look at their example suggests that the result is driven by the fact that in the Arrow-Debreu setting, markets are not reopened. Chattopadhyay and Gottardi (1999) consider differences between complete and sequentially complete market structures in overlapping generations models.

This paper studies multi-period economies subject to uncertainty, where markets may reopen at future states. At every state, households are endowed with preferences that may or may not be time-consistent. In general equilibrium models, time-inconsistent preferences have been introduced by Luttmer and Mariotti (2003) and Herings and Rohde (2006). We consider three market structures that are commonly believed to result in the same set of competitive equilibria. First, we consider the Arrow-Debreu (AD) structure with complete markets in the first period and no reopening of markets as time passes. Second, we consider sequentially complete (SC) markets, where in every state, people can trade the goods to be consumed in that state and all one-period ahead contingent commodities. Finally, we consider the complete (C) market structure. We say that markets are complete if, in every state, there are complete markets for all contingent commodities to be consumed in that state or in a later state. Figure 1 indicates which markets are open at various periods under these three market structures in an economy with four periods.

We follow Pollak (1968) in making a distinction between naïve and sophisticated behavior. Whereas naïve households believe that their preferences will not change in the future, sophisticated households correctly anticipate their future preferences. The latter households make plans that they expect to stick to in the future. Sophisticated households can be interpreted as consisting of multiple agents, playing a subgame perfect equilibrium against themselves. We will analyze these two types of behavior for both time-consistent and time-inconsistent preferences.

In general, the Arrow-Debreu market structure leads to equilibrium allocations that differ from equilibrium allocations corresponding to the other two market structures. The explanation is that the Arrow-Debreu market structure yields the commitment power needed not to re-trade. For sophisticated economies, sequentially complete markets yield the same allocations as complete markets do, even when preferences are allowed to be time-inconsistent. The assumption of sophisticated behavior is extremely 
Fig. 1 Commodities traded simultaneously in a four-period economy

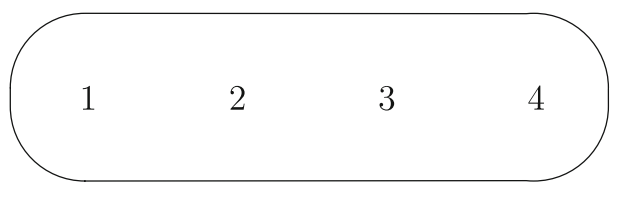

$\mathrm{AD}$

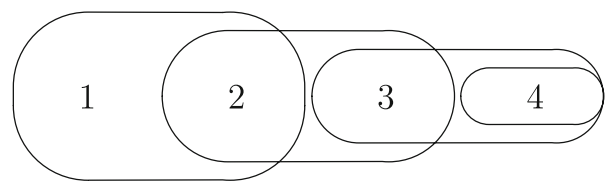

$\mathrm{SC}$

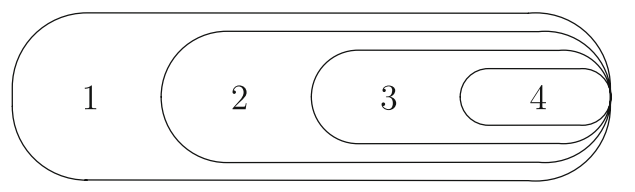

Fig. 2 Naïve equilibria when preferences are time-consistent

Fig. 3 Sophisticated equilibria when preferences are time-consistent
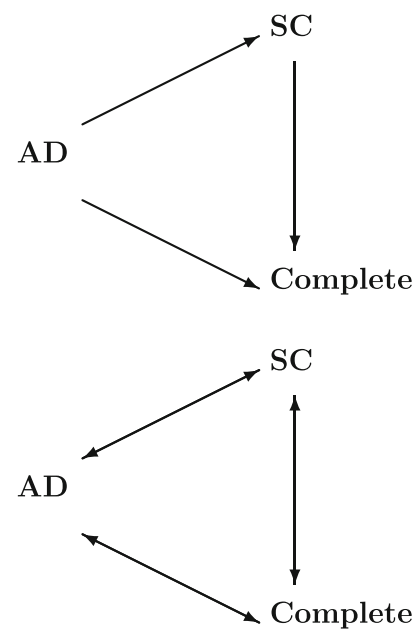

demanding. It might be more reasonable to assume that households, at least partly, mispredict their future preferences (Loewenstein et al. 2003). For naïve economies any sequentially complete equilibrium is allocationally equivalent to some complete equilibrium, but the converse statement does not hold.

When preferences are time-consistent, we show that all three markets structures are allocationally equivalent for sophisticated economies. For naïve economies, every equilibrium in the Arrow-Debreu market structure is allocationally equivalent to some sequentially complete equilibrium and some complete equilibrium, but surprisingly the reverse does not hold. The results of this paper are summarized in Figs. 2, 3, 4 and 5. In those figures, an arrow from ' $A$ ' to ' $B$ ' indicates that any concept ' $A$ ' equilibrium allocation is allocationally equivalent to some concept ' $\mathrm{B}$ ' equilibrium allocation.

This paper therefore identifies a crucial assumption that has to be satisfied in order for complete and sequentially complete markets to be equivalent: behavior should 
Fig. 4 Naïve equilibria when preferences are time-inconsistent
AD

SC

Fig. 5 Sophisticated equilibria when preferences are time-inconsistent

\section{更}

SC

AD

\section{Complete}

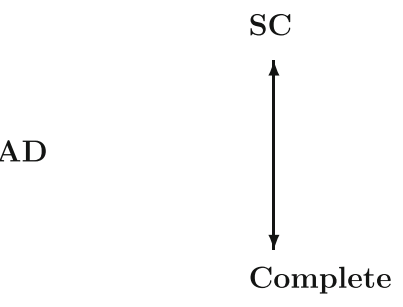

be sophisticated. For equivalence to Arrow-Debreu markets, it is also needed that preferences are time-consistent.

Section 2 starts by describing the primitives of the economies as considered in this paper, without elaborating on market structures. Section 3 describes the ArrowDebreu market structure. Sections 4 and 5 analyze the sequentially complete markets structure and the complete one, respectively. Both these sections are built up in the same fashion. First, naïve and sophisticated behavior is described. Then, properties of the specific market structure are studied. Section 6 then concludes. All proofs are in the Appendix.

\section{The primitives of the economy}

There is a finite number of periods, denoted by the integer $T .{ }^{1}$ In every period $t \in$ $\{2, \ldots, T\}$, exactly one event in the finite set $\Omega$ occurs. A sequence of $T-1$ events constitutes a state. We let $S=\Omega^{T-1}$ be the set of states. For every $s \in S$ and every $t \in\{2, \ldots, T\}$, we define the state at period $t$ by

$$
s(t)=\left\{\left(\omega_{\tau}\right)_{\tau=2}^{t} \in \Omega^{t-1} \mid \exists\left(\omega_{\tau}\right)_{\tau=t+1}^{T} \in \Omega^{T-t} \text { with }\left(\omega_{\tau}\right)_{\tau=2}^{T}=s\right\} .
$$

For $t=1$, we define $s(1)=1$.

Figure 6 illustrates the structure of an economy with four periods $(T=4)$ and two events in every period $(\Omega=\{\mathrm{Bad}, \mathrm{Good}\})$. A state in period 4 is a path from the root of the tree to an end-node. Thus, in the figure there are eight states in period 4 . Consider the state that corresponds to the path that leads to the third end-node from above. We call this state $s_{3}=(B, G, B)$. We have $s_{3}(2)=B, s_{3}(3)=(B, G)$, and $s_{3}(4)=$ $(B, G, B)$. For every $s(t)$ we define $s^{+}(t)=\left\{s^{\prime}(t+1) \in \Omega^{t} \mid s^{\prime} \in S, s^{\prime}(t)=s(t)\right\}$.

\footnotetext{
1 As no confusion will arise, for finite sets we will use the same notation to indicate the set and its cardinality.
} 
Fig. 6 An economy with $T=4$ and $\Omega=\{\mathrm{Bad}, \mathrm{Good}\}$

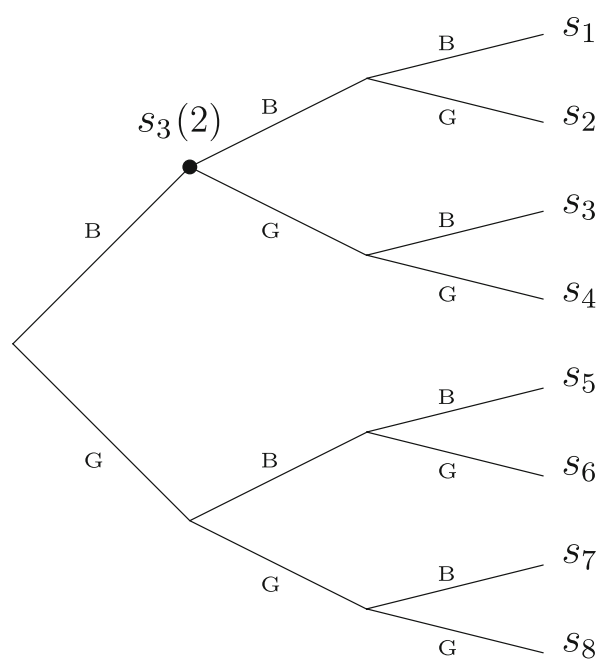

The economy consists of a finite number of households $h \in H$. In every state of every period, households consume a finite number of goods $l \in L$. For $s \in S$ and $\tau \geq t$, the consumption bundle $x_{s(\tau) \mid s(t)}^{h}$ denotes the consumption of every good at state $s(\tau)$ anticipated in state $s(t)$. Consumption for state $s(t)$ and every later state anticipated at $s(t)$, is given by the consumption plan $x_{\cdot \mid s(t)}^{h}$. Consumption for state $s(\tau)$ and all states that can be reached from there up till period $\tau^{\prime}$, as anticipated in state $s(t)$, is given by $x_{s(\tau), \tau^{\prime} \mid s(t)}^{h}$. By $x_{\cdot \mid s(t)}^{h} \imath \hat{x}_{\cdot \mid s(\tau)}^{h}$, we denote the consumption $x_{\cdot \mid s(t)}^{h}$ with $x_{s(\tau), T \mid s(t)}^{h}$ replaced by $\hat{x}_{\cdot \mid s(\tau)}^{h}$. For $t \in T$, we define $\mathcal{L}_{t}=L \sum_{\tau=1}^{T-t} \Omega^{\tau}$, the total number of commodities to be consumed at states later than $s(t)$. Consumption in every state is limited to the consumption sets $X_{\cdot \mid s(t)}^{h} \subset \mathbb{R}^{L+\mathcal{L}_{t}}$, where the interpretation of sub- and superscripts is similar as before. A subscript $l$ is added to denote consumption of good $l$. In every state $s(t)$, households have initial endowments $e_{\cdot \mid s(t)}^{h}$. Households are assumed to have correct expectations about consumption sets and endowments.

In every state, households have preferences over consumption in that state and all possible future states. Preferences of household $h$ in state $s(t)$ are represented by $\succeq^{h, s(t)}$, a relation defined on $X_{\cdot \mid s(t)}^{h}$. The preferences of household $h$ are denoted by $\succeq^{h}=\left(\succeq^{h, s(t)}\right)_{s \in S, t \in T}$.

Preferences might depend on the state of nature and can change over time. Consider a consumption plan in state $s(t)$. If we replace consumption from a future state $s(\tau)$ on by consumption that will be preferred once state $s(\tau)$ is reached, then time-consistency of preferences implies that the resulting consumption plan at state $s(t)$ is preferred to the original one.

Definition 2.1 (Time-consistent preferences) Preferences for household $h$ are timeconsistent if for all states $s \in S$, for all periods $t, \tau$ with $\tau \geq t$, for every $x_{\cdot \mid s(t)}^{h} \in X_{\cdot \mid s(t)}^{h}$ and every $\widehat{x}_{\cdot \mid s(\tau)}^{h} \in X_{\cdot \mid s(\tau)}^{h}$, we have

$$
x_{\cdot \mid s(t)}^{h} \succeq^{h, s(t)} x_{\cdot \mid s(t)}^{h} 2 \widehat{x}_{\cdot \mid s(\tau)}^{h}
$$


if and only if

$$
x_{s(\tau), T \mid s(t)}^{h} \succeq^{h, s(\tau)} \widehat{x}_{\cdot \mid s(\tau)}^{h} .
$$

Preferences are time-inconsistent if they are not time-consistent.

Preferences of a household in a particular state $s(t)$ are locally non-satiated in state $s\left(t^{\prime}\right)$, where $t^{\prime} \geq t$, if for every consumption plan in state $s(t)$, we can find another consumption plan in state $s(t)$ that (i) only deviates from the initial consumption plan in state $s\left(t^{\prime}\right)$, (ii) is arbitrarily close to the initial consumption plan, and (iii) is preferred to the initial consumption plan. This can be formalized as follows. Consider a household $h$, a state $s$, and two periods $t, t^{\prime}$ with $t^{\prime} \geq t$. Preferences $\succeq^{h, s(t)}$ are locally non-satiated in $s\left(t^{\prime}\right)$ if for every $x_{\cdot \mid s(t)}^{h} \in X_{\cdot \mid s(t)}^{h}$, for every $\varepsilon>0$, there is an $\bar{x}_{\cdot \mid s(t)}^{h} \in$ $X_{\cdot \mid s(t)}^{h}$ with $\bar{x}_{s^{\prime}(\tau) \mid s(t)}^{h}=x_{s^{\prime}(\tau) \mid s(t)}^{h}$ for every $s^{\prime}(\tau) \neq s\left(t^{\prime}\right),\left\|\bar{x}_{\cdot \mid s(t)}^{h}-x_{\cdot \mid s(t)}^{h}\right\|<\varepsilon$ and $\bar{x}_{\cdot \mid s(t)}^{h} \succ^{h, s(t)} x_{\cdot \mid s(t)}^{h}$. An economy with preferences $\left(\succeq^{h, s(t)}\right)_{h \in H, s \in S, t \in T}$ is locally non-satiated if for every state $s$ and every $t, t^{\prime}$ with $t^{\prime} \geq t$ there is a household $h$ such that $\succeq^{h, s(t)}$ is locally non-satiated in $s\left(t^{\prime}\right)$.

An economy $\mathcal{E}$ is described by its primitives $\left(X_{\cdot \mid 1}^{h}, e_{\cdot \mid 1}^{h}, \succeq^{h}\right)_{h \in H}$. A household will sell its endowments and use the revenues from this sale to buy the goods the household desires most. The timing of the opportunities to sell endowments will depend on the market structure.

\section{Arrow-Debreu markets}

This section considers the model of Arrow and Debreu (1954) and Debreu (1959). There are complete markets in contingent commodities in period 1. Markets do not reopen in later periods. All commodities can be traded in period 1 against the prices $p \cdot \mid 1$. Prices for commodities in state $s(t)$ are then given by $p_{s(t) \mid 1}$. Prices for state $s(t)$ and all states that can be reached from $s(t)$ are denoted by $p_{s(t), T \mid 1}$. We denote the set of possible prices by $P . \mid 1=\mathbb{R}^{L+\mathcal{L}_{1}}$.

In the Arrow-Debreu model, markets do not reopen after period 1. Therefore, it is implicitly assumed that consumption in those later periods is completely determined by the choices made in period 1 . Moreover, since markets do not reopen after period 1 , there is no need to specify prices in periods after period 1 . To be consistent in notation throughout this paper, we do want to specify those prices. Without loss of generality, we set prices in states later than period 1 equal to the prices in period 1 of corresponding contingent commodities. We define $p_{\cdot \mid s(t)}=p_{s(t), T \mid 1}$ for every $s(t)$, and $P \cdot \mid s(t)=\mathbb{R}^{L+\mathcal{L}_{t}}$ and $P=\prod_{s(t)} P \cdot \mid s(t)$.

The opportunity set of household $h$ in period 1 is given by

$$
\breve{\gamma}_{1}^{h}\left(p_{\cdot \mid 1}, e_{\cdot \mid 1}^{h}\right)=\left\{x_{\cdot \mid 1}^{h} \in X_{\cdot \mid 1}^{h} \mid p_{\cdot \mid 1} x_{\cdot \mid 1}^{h} \leq p_{\cdot \mid 1} e_{\cdot \mid 1}^{h}\right\} .
$$

The opportunity set of household $h$ in any state $s(t)$ with $t>1$ is given by

$$
\breve{\gamma}_{s(t)}^{h}\left(p_{\cdot \mid s(t)}, x_{s(t), T \mid s(t-1)}^{h}\right)=\left\{x_{s(t), T \mid s(t-1)}^{h}\right\} .
$$


A household selects a most preferred consumption plan in the opportunity set. Thus, the demand sets are given by

$$
\begin{aligned}
\breve{\delta}_{s(t)}^{h} & \left(p \cdot \mid s(t), x_{s(t), T \mid s(t-1)}^{h}\right)=\left\{\bar{x}_{\cdot \mid s(t)}^{h} \in \breve{\gamma}_{s(t)}^{h}\left(p_{\cdot \mid s(t)}, x_{s(t), T \mid s(t-1)}^{h}\right) \mid\right. \\
& \left.\nexists x_{\cdot \mid s(t)}^{h} \in \breve{\gamma}_{s(t)}^{h}\left(p \cdot \mid s(t), x_{s(t), T \mid s(t-1)}^{h}\right) \text { with } x_{\cdot \mid s(t)}^{h} \succ^{h, s(t)} \bar{x}_{\cdot \mid s(t)}^{h}\right\} .
\end{aligned}
$$

In equilibrium, total demand must equal total endowments. For notational purposes, we define $x_{1, T \mid s(0)}^{h}=e_{\cdot \mid 1}^{h}$.

Definition 3.1 (Arrow-Debreu (AD) equilibrium) A pair $\left(p^{*}, x^{*}\right) \in P \times X$ is an Arrow-Debreu $(A D)$ equilibrium of the economy $\mathcal{E}$ if

$$
\begin{aligned}
& \text { (a) } x_{\cdot \mid s(t)}^{* h} \in \breve{\delta}_{s(t)}^{h}\left(p_{\cdot \mid s(t)}^{*}, x_{s(t), T \mid s(t-1)}^{* h}\right) \text { for all } h \in H, t \in T, s \in S \text {, } \\
& \text { (b) } \sum_{h \in H} x_{\cdot \mid s(t)}^{* h}=\sum_{h \in H} e_{\cdot \mid s(t)}^{h} \text { for all } t \in T, s \in S, \\
& \text { (c) } p_{\cdot \mid s(t)}^{*}=p_{s(t), T \mid 1}^{*} \text { for all } t \in T, s \in S .
\end{aligned}
$$

\section{Sequentially complete markets}

This section considers sequentially complete markets. In every state there are spot markets and forward markets for one-period ahead contingent commodities. In period $t$ households can trade all goods for period $t$ and all goods for all possible events in period $t+1$. They have to form expectations about prices for goods that will be consumed in later periods, i.e., periods from $t+2 \mathrm{on}$. We assume that they form point expectations and that all households form the same expectations. We let $P \cdot \mid s(t)=\mathbb{R}^{L+\mathcal{L}_{t}}$, $\bar{Q}_{\cdot \mid s(t)}=\mathbb{R}^{\mathcal{L}_{t}}, P=\prod_{s(t)} P \cdot \mid s(t)$ and $\bar{Q}=\prod_{s(t)} \bar{Q}_{\cdot \mid s(t)}$. Prices and expected (at state $s(t))$ prices on spot markets will be denoted by $p_{\cdot \mid s(t)} \in P \cdot \mid s(t)$. Prices and expected (at state $s(t)$ ) prices of forward commodities will be denoted by $q_{\cdot \mid s(t)} \in \bar{Q}_{\cdot \mid s(t)}$. The (expected) prices in state $s(t)$ of commodities to be delivered in state $s(\tau)$ and traded in $s(\tau-1)$, are denoted by $q_{s(\tau) \mid s(t)}$. We define $\bar{Y}_{\cdot \mid s(t)}^{h}=\mathbb{R}^{\mathcal{L}_{t}}$ and $\bar{Y}=\prod_{h \in H} \prod_{s(t)} \bar{Y}_{\cdot \mid s(t)}^{h}$. For household $h$ the forward commodity bundle for state $s(\tau)$ that is expected in state $s(t)$ to be bought in state $s(\tau-1)$ is denoted by $y_{s(\tau) \mid s(t)}^{h}$. For notational purposes, we define $y_{\cdot \mid s(0)}^{h}=e_{\cdot \mid 1}^{h}$ and $y_{s(t) \mid s(t)}^{h}=y_{s(t) \mid s(t-1)}^{h}$.

The structure of sequentially complete markets is illustrated in Fig. 7. There, rows refer to the period in which the commodities are bought and columns refer to the period in which the commodities are delivered. The first block of four rows refers to the perspective of a household in period 1 . The next blocks are the perspectives of the households in the next periods. Bullets indicate that the prices and consumption are directly observable in the markets, open bullets denote expectations.

When markets can reopen in the future, and preferences are allowed to be timeinconsistent, a conflict can arise between the preferences from the perspective of one state and those of another, later, state. Following Pollak (1968), we make a distinction between naïve and sophisticated households. Naïve households will typically revise their plans over time. They have the incorrect belief that their preferences will not 
Fig. 7 Structure of sequentially complete markets

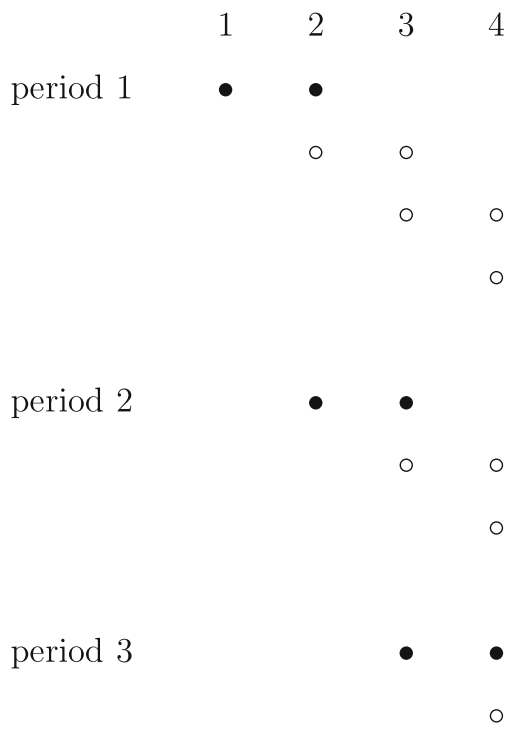

period 4

- - directly observable

$\circ-$ expectations

change in the future. Sophisticated households correctly anticipate their future preferences. They only make choices that they expect to stick to in the future. A more realistic assumption would be that households are partially sophisticated and incorrectly anticipate future preferences. For these households the relation between the various market structures will be similar to those for naïve households. Therefore, we restrict attention to naïve and (fully) sophisticated households. First, we consider naïve and then sophisticated behavior.

\subsection{Naïve behavior}

In every state, naïve households sell the endowments of that particular state and the one-period ahead endowments. They use the revenue from selling these commodities to buy goods on the spot markets and to buy one-period ahead contingent commodities. At each state $s(t)$ households form expectations about all future prices. We assume that naïve households have rational price expectations. By this we mean that in every period and every state they have common point expectations about prices and that prices are expected to be market clearing. Thus, rational price expectations are correct price expectations (Radner 1972) given the belief that preferences will not change in the future. Nevertheless, preferences can change and that will induce new rational 
price expectations in the future, which will again be correct from the viewpoint of that future state.

The opportunity set of household $h$ in state $s(t)$ is given by

$$
\begin{aligned}
& n \bar{\gamma}_{s(t)}^{h}\left(p \cdot|s(t), q \cdot| s(t), y_{s(t) \mid s(t-1)}^{h}, e_{t+1, T \mid s(t)}^{h}\right)=\left\{\left(x_{\cdot \mid s(t)}^{h}, y_{\cdot \mid s(t)}^{h}\right) \in X_{\cdot \mid s(t)}^{h} \times \bar{Y}_{\cdot \mid s(t)}^{h} \mid\right. \\
& p_{s^{\prime}(\tau) \mid s(t)} x_{s^{\prime}(\tau) \mid s(t)}^{h}+\sum_{s^{\prime}(\tau+1) \in s^{\prime+}(\tau)} q_{s^{\prime}(\tau+1) \mid s(t)} y_{s^{\prime}(\tau+1) \mid s(t)}^{h} \\
& \leq p_{s^{\prime}(\tau) \mid s(t)} y_{s^{\prime}(\tau) \mid s(t)}^{h}+\sum_{s^{\prime}(\tau+1) \in s^{\prime+}(\tau)} q_{s^{\prime}(\tau+1) \mid s(t)} e_{s^{\prime}(\tau+1) \mid s(t)}^{h} \\
& \left.\quad \text { for every } \tau \geq t, \text { for every } s^{\prime}(\tau) \text { with } s^{\prime}(t)=s(t)\right\},
\end{aligned}
$$

where, by definition, $y_{s(t) \mid s(t)}^{h}=y_{s(t) \mid s(t-1)}^{h}$ and $y_{1 \mid s(0)}^{h}=e_{1 \mid 1}^{h}$.

The demand set of household $h$ in state $s(t)$ is then given by

$$
\begin{aligned}
n \bar{\delta}_{s(t)}^{h} & \left(p_{\cdot \mid s(t)}, q \cdot \mid s(t), y_{s(t) \mid s(t-1)}^{h}, e_{t+1, T \mid s(t)}^{h}\right) \\
= & \left\{\left(\bar{x}_{\cdot \mid s(t)}^{h}, \bar{y}_{\cdot \mid s(t)}^{h}\right) \in{ }^{n} \bar{\gamma}_{s(t)}^{h}\left(p_{\cdot \mid s(t)}, q \cdot \mid s(t), y_{s(t) \mid s(t-1)}^{h}, e_{t+1, T \mid s(t)}^{h}\right) \mid\right. \\
& \nexists\left(x_{\cdot \mid s(t)}^{h}, y_{\cdot \mid s(t)}^{h}\right) \in{ }^{n} \bar{\gamma}_{s(t)}^{h}\left(p_{\cdot \mid s(t)}, q \cdot \mid s(t), y_{s(t) \mid s(t-1)}^{h}, e_{t+1, T \mid s(t)}^{h}\right) \\
& \text { with } \left.x_{\cdot \mid s(t)}^{h} \succ^{h, s(t)} \bar{x}_{\cdot \mid s(t)}^{h}\right\} .
\end{aligned}
$$

Prices and consumption are said to constitute a naïve sequentially complete equilibrium if consumption is in the demand sets and if markets clear and are expected to clear in every state.

Definition 4.1 (Naïve sequentially complete (SC) equilibrium) A pair ( $\left.p^{*}, q^{*}, x^{*}, y^{*}\right)$ $\in P \times \bar{Q} \times X \times \bar{Y}$ is a Nä̈ve sequentially complete (SC) equilibrium of the economy $\mathcal{E}$ if

(a) $\quad\left(x_{\cdot \mid s(t)}^{* h}, y_{\cdot \mid s(t)}^{* h}\right) \in n \bar{\delta}_{s(t)}^{h}\left(p_{\cdot \mid s(t)}^{*}, q_{\cdot \mid s(t)}^{*}, y_{s(t) \mid s(t-1)}^{* h}, e_{t+1, T \mid s(t)}^{h}\right)$ for all $h \in H$, $t \in T, s \in S$,

(b) $\quad \sum_{h \in H} x_{\cdot \mid s(t)}^{* h}=\sum_{h \in H} e_{\cdot \mid s(t)}^{h}$ for all $t \in T, s \in S$, and

(c) $\quad \sum_{h \in H} y_{\cdot \mid s(t)}^{* h}=\sum_{h \in H} e_{t+1, T \mid s(t)}^{h}$ for all $t \in T, s \in S$.

\subsection{Sophisticated behavior}

Sophisticated households know exactly how their preferences will change in the future. Therefore, they can correctly predict their future behavior. Moreover, they only make plans that they expect to adhere to. They face the additional constraint that their expected future consumption should be in their future demand sets given expected future prices. 
The opportunity set of sophisticated household $h$ in state $s(T)$ is the same as for its naïve counterpart:

$$
s \bar{\gamma}_{s(T)}^{h}\left(p_{\cdot \mid s(T)}, y_{s(T) \mid s(T-1)}^{h}\right)={ }^{n} \bar{\gamma}_{s(T)}^{h}\left(p_{\cdot \mid s(T)}, y_{s(T) \mid s(T-1)}^{h}\right) .
$$

The opportunity set of sophisticated household $h$ in state $s(t)$, where $t<T$, is given by

$$
\begin{aligned}
& s \bar{\gamma}_{s(t)}^{h}\left(p_{\cdot \mid s(t)}, q \cdot \mid s(t), y_{s(t) \mid s(t-1)}^{h}, e_{t+1, T \mid s(t)}^{h}\right)=\left\{\left(x_{\cdot \mid s(t)}^{h}, y_{\cdot \mid s(t)}^{h}\right) \in X_{\cdot \mid s(t)}^{h} \times \bar{Y}_{\cdot \mid s(t)}^{h} \mid\right. \\
& \quad(i) \quad p_{s^{\prime}(\tau) \mid s(t)} x_{s^{\prime}(\tau) \mid s(t)}^{h}+\sum_{s^{\prime}(\tau+1) \in s^{\prime+}(\tau)} q_{s^{\prime}(\tau+1) \mid s(t)} y_{s^{\prime}(\tau+1) \mid s(t)}^{h} \\
& \leq p_{s^{\prime}(\tau) \mid s(t)} y_{s^{\prime}(\tau) \mid s(t)}^{h}+\sum_{s^{\prime}(\tau+1) \in s^{\prime+}(\tau)} q_{s^{\prime}(\tau+1) \mid s(t)} e_{s^{\prime}(\tau+1) \mid s(t)}^{h}
\end{aligned}
$$

for every $\tau \geq t$, for every $s^{\prime}(\tau)$ with $s^{\prime}(t)=s(t)$,

and

$$
\text { (ii) } \quad\left(\begin{array}{rl}
\left.x_{s(t+1), T \mid s(t)}^{h}, y_{s^{+}(t+1), T \mid s(t)}^{h}\right) \in \\
\quad s \bar{\delta}_{s(t+1)}^{h}\left(p_{s(t+1), T \mid s(t)}, q_{s^{+}(t+1), T \mid s(t)}, y_{s(t+1) \mid s(t)}^{h}, e_{t+2, T \mid s(t+1)}^{h}\right) \\
\left.\quad \text { for every } s(t+1) \in s^{+}(t)\right\} .
\end{array}\right.
$$

The demand set of a sophisticated household is given by

$$
\begin{aligned}
s \bar{\delta}_{s(t)}^{h}( & \left.p \cdot|s(t), q \cdot| s(t), y_{s(t) \mid s(t-1)}^{h}, e_{t+1, T \mid s(t)}^{h}\right) \\
= & \left\{\left(\bar{x}_{\cdot \mid s(t)}^{h}, \bar{y}_{\cdot \mid s(t)}^{h}\right) \in s \bar{\gamma}_{s(t)}^{h}\left(p_{\cdot \mid s(t)}, q \cdot \mid s(t), y_{s(t) \mid s(t-1)}^{h}, e_{t+1, T \mid s(t)}^{h}\right) \mid\right. \\
& \nexists\left(x_{\cdot \mid s(t)}^{h}, y_{\cdot \mid s(t)}^{h}\right) \in{ }^{s} \bar{\gamma}_{s(t)}^{h}\left(p_{\cdot \mid s(t)}, q \cdot \mid s(t), y_{s(t) \mid s(t-1)}^{h}, e_{t+1, T \mid s(t)}^{h}\right) \\
& \text { with } \left.x_{\cdot \mid s(t)}^{h} \succ^{h, s(t)} \bar{x}_{\cdot \mid s(t)}^{h}\right\} .
\end{aligned}
$$

Prices, consumption bundles and purchases of forward commodities constitute a sophisticated sequentially complete equilibrium if actual and planned supply of spot and forward commodities equals actual and planned demand, price expectations are correct, and expected consumption and expected purchases of forward commodities equal realized consumption and realized purchases of forward commodities. This is formalized in the following definition.

Definition 4.2 (Sophisticated sequentially complete (SC) equilibrium) A pair ( $p^{*}, q^{*}$, $\left.x^{*}, y^{*}\right) \in P \times \bar{Q} \times X \times \bar{Y}$ is a Sophisticated sequentially complete (SC) equilibrium of the economy $\mathcal{E}$ if

(a) $\quad\left(x_{\cdot \mid s(t)}^{* h}, y_{\cdot \mid s(t)}^{* h}\right) \in s \bar{\delta}_{s(t)}^{h}\left(p_{\cdot \mid s(t)}^{*}, q_{\cdot \mid s(t)}^{*}, y_{s(t) \mid s(t-1)}^{* h}, e_{t+1, T \mid s(t)}^{h}\right)$ for all $h \in H$, $t \in T, s \in S$, 
(b) $\quad \sum_{h \in H} x_{\cdot \mid s(t)}^{* h}=\sum_{h \in H} e_{\cdot \mid s(t)}^{h}$ for all $t \in T, s \in S$,

(c) $\sum_{h \in H} y_{\cdot \mid s(t)}^{* h}=\sum_{h \in H} e_{t+1, T \mid s(t)}^{h}$ for all $t \in T, s \in S$,

(d) $p_{s(\tau), T \mid s(t)}^{*}=p_{\cdot \mid s(\tau)}^{*}$ for all $s \in S, t, \tau \in T$ with $t \leq \tau$,

(e) $q_{s(\tau), T \mid s(t)}^{*}=q_{s(\tau), T \mid s(\tau-1)}^{*}$ for all $s \in S, t, \tau \in T$ with $t<\tau$,

(f) $\quad x_{s(\tau), T \mid s(t)}^{* h}=x_{\cdot \mid s(\tau)}^{* h}$ for all $h \in H, s \in S, t, \tau \in T$ with $t \leq \tau$, and

(g) $\quad y_{s(\tau), T \mid s(t)}^{* h}=y_{s(\tau), T \mid s(\tau-1)}^{* h}$ for all $h \in H, s \in S, t, \tau \in T$ with $t<\tau$.

\subsection{Properties of sequentially complete equilibria}

In both naïve SC and sophisticated SC equilibria, expectations of relative prices on spot markets are equal to the corresponding expected relative prices of assets or oneperiod ahead contingent commodities, except when all those asset prices are equal to zero. This is formalized in the following lemma. Svensson (1976) derives a similar result in a two-period economy without uncertainty. He called these price expectations stationary point expectations.

Theorem 4.3 Let the economy $\mathcal{E}$ be locally non-satiated and let $\left(p^{*}, q^{*}, x^{*}, y^{*}\right)$ be a naïve $S C$ equilibrium of $\mathcal{E}$. Then for every $s, s^{\prime} \in S$ and every $t, \tau \in T$ with $\tau>t$ and $s^{\prime}(t)=s(t)$ there is $\mu_{s^{\prime}(\tau) \mid s(t)} \in \mathbb{R}$ such that

$$
q_{S^{\prime}(\tau) \mid s(t)}^{*}=\mu_{s^{\prime}(\tau) \mid s(t)} p_{s^{\prime}(\tau) \mid s(t)}^{*}
$$

The result of Theorem 4.3 is driven by the arbitrage opportunities that are created in locally non-satiated economies if the condition of the result would be violated. This intuition is entirely correct for the naïve case. The sophisticated case is more difficult. The reason is that a sophisticated household cannot guarantee that his future self will behave as he would like him to behave. Therefore, changing income in a future state may make the future self consume a bundle that the current self does not like at all. The availability of an arbitrage opportunity in a particular state is not sufficient to drive the result. The arbitrage opportunity needs to be such that also income in future states is unchanged, so that future selves will behave the same, irrespective of whether the arbitrage opportunity is taken or not. Our proof for the naïve case is constructed in such a way that these properties are satisfied, and applies therefore to the sophisticated case as well.

Theorem 4.4 Let the economy $\mathcal{E}$ be locally non-satiated and let $\left(p^{*}, q^{*}, x^{*}, y^{*}\right)$ be a sophisticated $S C$ equilibrium of $\mathcal{E}$. Then for every $s, s^{\prime} \in S$ and every $t, \tau \in T$ with $\tau>t$ and $s^{\prime}(t)=s(t)$ there is a $\mu_{s^{\prime}(\tau) \mid s(t)} \in \mathbb{R}$ such that

$$
q_{s^{\prime}(\tau) \mid s(t)}^{*}=\mu_{s^{\prime}(\tau) \mid s(t)} p_{s^{\prime}(\tau) \mid s(t)}^{*}
$$

An $\mathrm{AD}$ equilibrium $\left(p^{*}, q^{*}\right)$ is said to be allocationally equivalent to a naïve (sophisticated) SC equilibrium if there are $p \in P, q \in \bar{Q}$ and $y \in \bar{Y}$ such that $\left(p, q, x^{*}, y\right)$ is a naïve (sophisticated) SC equilibrium. Similarly, a naïve (sophisticated) SC equilibrium $\left(p^{*}, q^{*}, x^{*}, y^{*}\right)$ is said to be allocationally equivalent to an $\mathrm{AD}$ equilibrium if there is $p \in P$ such that $\left(p, x^{*}\right)$ is an $\mathrm{AD}$ equilibrium. 
When preferences are time-consistent one might expect that there is no difference between naïve and sophisticated behavior. However, this is not the case. We show by means of an example that naïve SC equilibria are not necessarily allocationally equivalent to $\mathrm{AD}$ equilibria. However, sophisticated SC equilibria are allocationally equivalent to $\mathrm{AD}$ equilibria and the other way around. The difference arises because in an $\mathrm{AD}$ equilibrium price expectations are correct, whereas in a naïve SC equilibrium price expectations are only rational. The key insight used in the example is that even in the time-consistent case, rational price expectations need not be correct. Finally, every $\mathrm{AD}$ equilibrium is allocationally equivalent to some $\mathrm{SC}$ equilibrium in both the naïve and the sophisticated case. These results will be derived next.

The following example with time-consistent preferences displays naïve SC equilibria that are not allocationally equivalent to any AD equilibrium. It is borrowed from Drèze and Herings (2003). Hellwig (1983) considered a similar example for the case with incomplete markets.

Example 4.5 Consider a two-period economy without uncertainty. There are two agents and two goods in every period. Endowments are $e_{\cdot \mid 1}^{h}=(2,2,2,2)$ for $h=1,2$. Preferences are time-consistent and represented by

$$
u_{1}^{h}\left(x_{\cdot \mid 1}^{h}\right)=v^{h}\left(x_{1,1 \mid 1}^{h}, x_{1,2 \mid 1}^{h}\right)^{\frac{1}{2}} \cdot v^{h}\left(x_{2,1 \mid 1}^{h}, x_{2,2 \mid 1}^{h}\right)^{\frac{1}{2}}
$$

where

$$
v^{1}\left(x_{\cdot, 1 \mid 1}^{1}, x_{\cdot, 2 \mid 1}^{1}\right)= \begin{cases}{\left[\min \left(x_{\cdot, 1 \mid 1}^{1}, x_{\cdot, 2 \mid 1}^{1}\right)\right],} & \min \left(x_{\cdot, 1 \mid 1}^{1}, x_{\cdot, 2 \mid 1}^{1}\right) \leq 1, \\ {\left[\left(x_{\cdot, 1 \mid 1}^{1}-1\right)^{\frac{1}{2}}\left(x_{\cdot, 2 \mid 1}^{1}-1\right)^{\frac{1}{2}}+1\right],} & \min \left(x_{\cdot, 1 \mid 1}^{1}, x_{\cdot, 2 \mid 1}^{1}\right) \geq 1,\end{cases}
$$

and

$$
v^{2}\left(x_{\cdot, 1 \mid 1}^{2}, x_{\cdot, 2 \mid 1}^{2}\right)= \begin{cases}{\left[\min \left(x_{\cdot, 1 \mid 1}^{2}, x_{\cdot, 2 \mid 1}^{2}\right)\right],} & \min \left(x_{\cdot, 1 \mid 1}^{2}, x_{\cdot, 2 \mid 1}^{2}\right) \leq 3 \\ {\left[\left(x_{\cdot, 1 \mid 1}^{2}-3\right)^{\frac{1}{2}}\left(x_{\cdot, 2 \mid 1}^{2}-3\right)^{\frac{1}{2}}+3\right],} & \min \left(x_{\cdot, 1 \mid 1}^{2}, x_{\cdot, 2 \mid 1}^{2}\right) \geq 3 .\end{cases}
$$

Now consider sequentially complete markets. Let $p_{. \mid 1}^{*}=(1 / 2,1 / 2,1 / 2,1 / 2)$, $q_{2 \mid 1}^{*}=(1 / 2,1 / 2), p_{\cdot \mid 2}^{*}=(3 / 4,1 / 4)$. Consider the following allocation

$$
\begin{array}{ll}
x_{\cdot \mid 1}^{* 1}=(2,2,2,2) & x_{\cdot \mid 1}^{* 2}=(2,2,2,2) \\
x_{\cdot \mid 2}^{* 1}=(1,1) & x_{\cdot \mid 2}^{* 2}=(3,3)
\end{array}
$$

achieved by the asset allocations

$$
\begin{aligned}
& y_{2 \mid 1}^{* 1}=(0,4), \\
& y_{2 \mid 1}^{* 2}=(4,0) .
\end{aligned}
$$


It can be verified that $\left(p^{*}, q^{*}, x^{*}, y^{*}\right)$ is a naïve $\mathrm{SC}$ equilibrium. In particular it holds that the choice of $y_{2 \mid 1}^{* 1}=(0,4)$ and $y_{2 \mid 1}^{* 2}=(4,0)$ is optimal given prices and price expectations $p_{\cdot \mid 1}^{*}=(1 / 2,1 / 2,1 / 2,1 / 2)$ and it holds that for the subeconomy starting in period 2 with initial endowments $y_{2 \mid 1}^{* 1}=(0,4)$ and $y_{2 \mid 1}^{* 2}=(4,0)$, prices $p_{. \mid 2}^{*}=(3 / 4,1 / 4)$ constitute a competitive equilibrium. The prices for contingent commodities in period 1 are not proportional to actual prices in period 2 . Thus, in period 1 households have rational expectations about prices in period 2, but in period 2 they are confronted with different competitive equilibrium prices. Since $x_{\cdot \mid 1}^{1}=(2,2,1,1)$ is not individually rational for household 1 from the perspective of period 1 , there can be no $p$ such that $(p,(2,2,1,1),(2,2,3,3))$ is an $\mathrm{AD}$ equilibrium.

When preferences are time-consistent, every $\mathrm{AD}$ equilibrium is allocationally equivalent to some naïve and to some sophisticated SC equilibrium. This is shown in the following theorems. Crucial in the derivation of the first result is that the set of correct price expectations is a subset of the set of rational price expectations.

Theorem 4.6 Let preferences be time-consistent and let the economy $\mathcal{E}$ be locally non-satiated. Let $\left(p^{*}, x^{*}\right)$ be an $A D$ equilibrium of $\mathcal{E}$. Then there is $\left(q^{*}, y^{*}\right) \in \bar{Q} \times \bar{Y}$ such that $\left(p^{*}, q^{*}, x^{*}, y^{*}\right)$ is a naïve $S C$ equilibrium of $\mathcal{E}$.

Theorem 4.7 Let preferences be time-consistent and let the economy $\mathcal{E}$ be locally non-satiated. Let $\left(p^{*}, x^{*}\right)$ be an $A D$ equilibrium of $\mathcal{E}$. Then there is $\left(q^{*}, y^{*}\right) \in \bar{Q} \times \bar{Y}$ such that $\left(p^{*}, q^{*}, x^{*}, y^{*}\right)$ is a sophisticated $S C$ equilibrium of $\mathcal{E}$.

Every sophisticated SC equilibrium is allocationally equivalent to some AD equilibrium if preferences are time-consistent, as is shown next. The fact that sophisticated households have correct price expectations is important for deriving this result. Notice that we need to make some additional assumptions for this direction, because we need optimal consumption plans to exist in the opportunity sets. The preferences of household $h$ are said to be acyclic if for every state $s(t)$ there is no finite set $\left\{x_{\cdot \mid s(t)}^{h, 1}, \ldots, x_{\cdot \mid s(t)}^{h, n}\right\} \subset X_{\cdot \mid s(t)}^{h}$ such that $x_{\cdot \mid s(t)}^{h, i} \succ^{h, s(t)} x_{\cdot \mid s(t)}^{h, i+1}$ for $i=1, \ldots, n-1$ and such that $x_{\cdot \mid s(t)}^{h, n} \succ^{h, s(t)} x_{\cdot \mid s(t)}^{h, 1}$. The preferences of household $h$ are said to have open lower sections if for every state $s(t)$, for every $x_{\cdot \mid s(t)}^{h} \in X_{\cdot \mid s(t)}^{h}$, the set $\left\{\widehat{x}_{\cdot \mid s(t)}^{h} \in X_{\cdot \mid s(t)}^{h} \mid\right.$ $\left.x_{\cdot \mid s(t)}^{h} \succ^{h, s(t)} \hat{x}_{\cdot \mid s(t)}^{h}\right\}$ is open in $X_{\cdot \mid s(t)}^{h}$.

Theorem 4.8 Let preferences be time-consistent, locally non-satiated, acyclic, and have open lower sections, and let consumption sets be closed and bounded from below. Let $\left(p^{*}, q^{*}, x^{*}, y^{*}\right)$ be a sophisticated $S C$ equilibrium of $\mathcal{E}$ with $p^{*} \gg 0$. Then there is a $p$ such that $\left(p, x^{*}\right)$ is an $A D$ equilibrium of $\mathcal{E}$.

In general, when preferences might be time-inconsistent, there is no relation between $\mathrm{AD}$ equilibria and naïve or sophisticated SC equilibria. When preferences are timeinconsistent conflicts arise between current and future preferences. A naïve household will typically deviate from planned consumption, since he does not anticipate a change in his future preferences. Sophisticated households do anticipate future changes, and are therefore constrained by their future behavior. On the contrary, the AD market setting ensures perfect commitment in period 1, which is optimal from the perspective 
of the preferences of households in period 1. Constraints coming from the behavior of future selves are thereby irrelevant.

\section{Complete markets}

In this section, there are complete markets for all possible contingent commodities at every state of every period. Again, $p_{\cdot \mid s(t)}$ denotes the expected (at state $s(t)$ ) prices on the spot markets. In state $s(t)$, the expected state $s^{\prime}(\tau)$ prices for contingent commodities that are delivered in state $s^{\prime \prime}(\tilde{\tau})$ are given by $q_{\left(s^{\prime \prime}(\tilde{\tau}) \mid s^{\prime}(\tau)\right) \mid s(t)}$, where $\tilde{\tau}>\tau \geq t$, $s^{\prime \prime}(\tau)=s^{\prime}(\tau)$, and $s^{\prime}(t)=s(t)$. The expectations in state $s(t)$ of prices for all 'future' contingent commodities that can possibly be traded from state $s^{\prime}(\tau)$ on, are denoted by $q_{\left(\cdot \mid s^{\prime}(\tau), T\right) \mid s(t)}$. We define $Q_{\cdot \mid s(t)}=\mathbb{R}^{\sum_{\tilde{\tau}=t}^{T-1} \mathcal{L}_{\tilde{\tau}}}$. We let $y_{\left(s^{\prime}(\tilde{\tau}) \mid s^{\prime}(\tau)\right) \mid s(t)}^{h}$ denote bundles of contingent commodities that are expected at $s(t)$ to be bought in $s^{\prime}(\tau)$ and that are delivered in $s^{\prime}(\tilde{\tau})$. For notational purposes, we define $y_{(\cdot \mid s(0)) \mid s(0)}^{h}=e_{\cdot \mid 1}^{h}$. We also define $Y_{\cdot \mid s(t)}^{h}=\mathbb{R}^{\sum_{\tilde{\tau}=t}^{T-1} \mathcal{L}_{\tilde{\tau}}}$. Finally,

$$
\begin{aligned}
X & =\prod_{h \in H} \prod_{s(t)} X_{\cdot \mid s(t)}^{h}, \quad Y=\prod_{h \in H} \prod_{s(t)} Y_{\cdot \mid s(t)}^{h}, \quad P=\prod_{s(t)} P_{\cdot \mid s(t)}, \\
Q & =\prod_{s(t)} Q_{\cdot \mid s(t)} .
\end{aligned}
$$

Figure 8 illustrates the structure of complete markets and is analogous to Fig. 7.

\subsection{Naïve behavior}

In every state households sell their endowments which are determined by previous purchases of contingent commodities. With the revenues of these sales they buy goods on spot markets and contingent commodities on asset markets. The contingent commodities bought determine the endowments at future states. In order to make a choice, a household needs to have expectations about prices of goods and contingent commodities in the future so as to know what to buy. As before, naïve households have rational price expectations. Thus, they all have the same point expectations about prices on spot and forward-commodity markets and prices are expected to be market clearing. We will show later that, in equilibrium, households cannot expect prices to change a lot, since that would make them believe that there are arbitrage opportunities, so that demand of some commodities would be infinite. In every period households form new rational price expectations. The new expectations need not be equal to the expectations from the period before. This again is due to the changing preferences. This does not contradict with the fact that previous expectations were rational, since naïve households always have the incorrect belief that their preferences will not change. Again, rational price expectations are correct price expectations given this belief. The opportunity set of household $h$ in state $s(t)$ is given by 
Fig. 8 Structure of complete markets $\begin{array}{ccccc} & 1 & 2 & 3 & \\ \text { period } 1 & \bullet & \bullet & \bullet & \\ & \circ & \circ & \circ \\ & & \circ & \circ\end{array}$

4

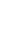

period 2

period 3

period 4

- - directly observable

- - expectations

$$
\begin{aligned}
& { }^{n} \gamma_{s(t)}^{h}\left(p_{\cdot \mid s(t)}, q \cdot \mid s(t), y_{(\cdot \mid s(t-1)) \mid s(t-1)}^{h}\right)=\left\{\left(x_{\cdot \mid s(t)}^{h}, y_{\cdot \mid s(t)}^{h}\right) \in X_{\cdot \mid s(t)}^{h} \times Y_{\cdot \mid s(t)}^{h} \mid\right. \\
& p_{s^{\prime}(\tau) \mid s(t)} x_{s^{\prime}(\tau) \mid s(t)}^{h}+q_{\left(\cdot \mid s^{\prime}(\tau)\right) \mid s(t)} y_{\left(\cdot \mid s^{\prime}(\tau)\right) \mid s(t)}^{h} \\
& \leq p_{s^{\prime}(\tau) \mid s(t)} y_{\left(s^{\prime}(\tau) \mid s^{\prime}(\tau-1)\right) \mid s(t)}^{h}+q_{\left(\cdot \mid s^{\prime}(\tau)\right) \mid s(t)} y_{\left(s^{\prime+}(\tau), T \mid s^{\prime}(\tau-1)\right) \mid s(t)}^{h} \\
& \text { for every } \left.\tau \geq t \text {, and every } s^{\prime}(\tau) \text { with } s^{\prime}(t)=s(t)\right\} \text {, }
\end{aligned}
$$

where, by definition, $y_{(\cdot \mid s(0)) \mid s(0)}^{h}=e_{\cdot \mid 1}^{h}$ and $y_{(\cdot \mid s(t-1)) \mid s(t)}^{h}=y_{(\cdot \mid s(t-1)) \mid s(t-1)}^{h}$. The demand set is then given by

$$
\begin{aligned}
& { }^{n} \delta_{s(t)}^{h}\left(p_{\cdot \mid s(t)}, q_{\cdot \mid s(t)}, y_{(\cdot \mid s(t-1)) \mid s(t-1)}^{h}\right) \\
& =\left\{\left(\bar{x}_{\cdot \mid s(t)}^{h}, \bar{y}_{\cdot \mid s(t)}^{h}\right) \in{ }^{n} \gamma_{s(t)}^{h}\left(p_{\cdot \mid s(t)}, q_{\cdot \mid s(t)}, y_{(\cdot \mid s(t-1)) \mid s(t-1)}^{h}\right) \mid\right. \\
& \quad \nexists\left(x_{\cdot \mid s(t)}^{h}, y_{\cdot \mid s(t)}^{h}\right) \in{ }^{n} \gamma_{s(t)}^{h}\left(p_{\cdot \mid s(t)}, q_{\cdot \mid s(t)}, y_{(\cdot \mid s(t-1)) \mid s(t-1)}^{h}\right) \\
& \left.\quad \text { with } x_{\cdot \mid s(t)}^{h} \succ^{h, s(t)} \bar{x}_{\cdot \mid s(t)}^{h}\right\} .
\end{aligned}
$$

In equilibrium all markets should clear and should be expected to clear. 
Definition 5.1 (Naïve complete equilibrium) A pair $\left(p^{*}, q^{*}, x^{*}, y^{*}\right) \in P \times Q \times X \times Y$ is a Nä̈ve Complete equilibrium of the economy $\mathcal{E}$ if
(a) $\quad\left(x_{\cdot \mid s(t)}^{* h}, y_{\cdot \mid s(t)}^{* h}\right) \in{ }^{n} \delta_{s(t)}^{h}\left(p_{\cdot \mid s(t)}^{*}, q_{\cdot \mid s(t)}^{*}, y_{(\cdot \mid s(t-1)) \mid s(t-1)}^{* h}\right)$ for all $h \in H, s \in S$, $t \in T$,
(b) $\quad \sum_{h \in H} x_{\cdot \mid s(t)}^{* h}=\sum_{h \in H} e_{\cdot \mid s(t)}^{h}$ for all $s \in S, t \in T$, and
(c) $\quad \sum_{h \in H} y_{(\cdot \mid s(\tau)) \mid s(t)}^{* h}=\sum_{h \in H} e_{\tau+1, T \mid s(\tau)}^{h}$ for all $s \in S, t, \tau \in T$ with $t \leq \tau$.

\subsection{Sophisticated behavior}

In period $T$ the opportunity set of a sophisticated household $h$ in state $s$ is the same as the opportunity set for its naïve counterpart, i.e.,

$$
{ }^{s} \gamma_{s(T)}^{h}\left(p \cdot \mid s(T), y_{\cdot \mid s(T-1)}^{h}\right)={ }^{n} \gamma_{s(T)}^{h}\left(p \cdot \mid s(T), y_{\cdot \mid s(T-1)}^{h}\right) .
$$

The opportunity set of a sophisticated household $h$ in state $s(t)$, where $t<T$, is given by

$$
\begin{aligned}
& { }^{s} \gamma_{s(t)}^{h}\left(p_{\cdot \mid s(t)}, q \cdot \mid s(t), y_{(\cdot \mid s(t-1)) \mid s(t-1)}^{h}\right)=\left\{\left(x_{\cdot \mid s(t)}^{h}, y_{\cdot \mid s(t)}^{h}\right) \in X_{\cdot \mid s(t)}^{h} \times Y_{\cdot \mid s(t)}^{h} \mid\right. \\
& \quad(i) \quad p_{s^{\prime}(\tau) \mid s(t)} x_{s^{\prime}(\tau) \mid s(t)}^{h}+q_{\left(\cdot \mid s^{\prime}(\tau)\right) \mid s(t)} y_{\left(\cdot \mid s^{\prime}(\tau)\right) \mid s(t)}^{h} \\
& \leq p_{s^{\prime}(\tau) \mid s(t)} y_{\left(s^{\prime}(\tau) \mid s^{\prime}(\tau-1)\right) \mid s(t)}^{h}+q_{\left(\cdot \mid s^{\prime}(\tau)\right) \mid s(t)} y_{\left(s^{\prime}(\tau), T \mid s^{\prime}(\tau-1)\right) \mid s(t)}^{h} \\
& \quad \text { for every } \tau \geq t, \text { and every } s^{\prime}(\tau) \text { with } s^{\prime}(t)=s(t),
\end{aligned}
$$

and

$$
\begin{aligned}
& \text { (ii) } \quad\left(x_{s(t+1), T \mid s(t)}^{h}, y_{(\cdot \mid s(t+1), T) \mid s(t)}^{h}\right) \in \\
& { }^{s} \delta_{s(t+1)}^{h}\left(p_{s(t+1), T \mid s(t)}, q_{(\cdot \mid s(t+1), T) \mid s(t)}, y_{(\cdot \mid s(t)) \mid s(t)}^{h}\right) \\
& \left.\quad \text { for every } s(t+1) \in s^{+}(t)\right\} .
\end{aligned}
$$

The demand set of a sophisticated household is as follows.

$$
\begin{aligned}
{ }^{s} \delta_{s(t)}^{h} & \left(p_{\cdot \mid s(t)}, q_{\cdot \mid s(t)}, y_{(\cdot \mid s(t-1)) \mid s(t-1)}^{h}\right) \\
= & \left\{\left(\bar{x}_{\cdot \mid s(t)}^{h}, \bar{y}_{\cdot \mid s(t)}^{h}\right) \in{ }^{s} \gamma_{s(t)}^{h}\left(p_{\cdot \mid s(t)}, q_{\cdot \mid s(t)}, y_{(\cdot \mid s(t-1)) \mid s(t-1)}^{h}\right) \mid\right. \\
& \nexists\left(x_{\cdot \mid s(t)}^{h}, y_{\cdot \mid s(t)}^{h}\right) \in{ }^{s} \gamma_{s(t)}^{h}\left(p_{\cdot \mid s(t)}, q \cdot \mid s(t), y_{(\cdot \mid s(t-1)) \mid s(t-1)}^{h}\right) \\
& \text { with } \left.x_{\cdot \mid s(t)}^{h} \succ^{h, s(t)} \bar{x}_{\cdot \mid s(t)}^{h}\right\} .
\end{aligned}
$$

Finally, we define a sophisticated complete equilibrium as follows. Note that the difference with a naïve complete equilibrium is that sophisticated households have correct price expectations and correct expectations about future purchases. 
Definition 5.2 (sophisticated complete equilibrium) A pair $\left(p^{*}, q^{*}, x^{*}, y^{*}\right) \in P \times$ $Q \times X \times Y$ is a sophisticated complete equilibrium of the economy $\mathcal{E}$ if $\left(x_{\cdot \mid s(t)}^{* h}, y_{\cdot \mid s(t)}^{* h}\right) \in{ }^{s} \delta_{s(t)}^{h}\left(p_{\cdot \mid s(t)}^{*}, q_{\cdot \mid s(t)}^{*}, y_{(\cdot \mid s(t-1)) \mid s(t-1)}^{* h}\right)$ for all $h \in H, s \in S$, $t \in T$,

(b) $\quad \sum_{h \in H} x_{\cdot \mid s(t)}^{* h}=\sum_{h \in H} e_{\cdot \mid s(t)}^{h}$ for all $s \in S, t \in T$,

(c) $\sum_{h \in H} y_{(\cdot \mid s(\tau)) \mid s(t)}^{* h}=\sum_{h \in H} e_{\tau+1, T \mid s(\tau)}^{h}$ for all $s \in S, t, \tau \in T$ with $t \leq \tau$,

(d) $p_{s(\tau), T \mid s(t)}^{*}=p_{\cdot \mid s(\tau)}^{*}$ for all $s \in S, t, \tau \in T$ with $t \leq \tau$,

(e) $\quad q_{(\cdot \mid s(\tau), T) \mid s(t)}^{*}=q_{\cdot \mid s(\tau)}^{*}$ for all $s \in S, t, \tau \in T$ with $t \leq \tau$,

(f) $\quad x_{s(\tau), T \mid s(t)}^{* h}=x_{\cdot \mid s(\tau)}^{* h}$ for all $h \in H, s \in S, t, \tau \in T$ with $t \leq \tau$, and

(g) $\quad y_{(\cdot \mid s(\tau), T) \mid s(t)}^{* h}=y_{\cdot \mid s(\tau)}^{* h}$ for all $h \in H, s \in S, t, \tau \in T$ with $t \leq \tau$.

\subsection{Properties of complete equilibria}

The essential difference between complete (C) and sequentially complete (SC) markets is that in complete markets there is a richer set of assets available. As a consequence, in a three-period example for instance, under $\mathrm{C}$ it is possible in period 1 to make binding commitments regarding the delivery and purchases of commodities in periods 2 and 3. When price expectations regarding periods 2 and 3 do not change, this is inconsequential. All that matters is available wealth in each period, and not the composition of wealth, i.e. the distribution of wealth over the various assets. When price expectations do change, different compositions of wealth typically do have real consequences. Under SC there is less choice for the composition of wealth. In this section we will therefore address the question of how complete and sequentially complete equilibria relate to one another.

An $\mathrm{AD}$ equilibrium $\left(p^{*}, q^{*}\right)$ is said to be allocationally equivalent to a naïve (sophisticated) complete equilibrium if there are $p \in P, q \in Q$ and $y \in Y$ such that $\left(p, q, x^{*}, y\right)$ is a naïve (sophisticated) complete equilibrium. Similarly, a naïve (sophisticated) complete equilibrium $\left(p^{*}, q^{*}, x^{*}, y^{*}\right)$ is said to be allocationally equivalent to an $\mathrm{AD}$ equilibrium if there is a $p \in P$ such that $\left(p, x^{*}\right)$ is an $\mathrm{AD}$ equilibrium. A naïve (sophisticated) complete equilibrium $\left(p^{*}, q^{*}, x^{*}, y^{*}\right)$ is said to be allocationally equivalent to a naïve (sophisticated) SC equilibrium if there are $p \in P, q \in \bar{Q}$ and $y \in \bar{Y}$ such that $\left(p, q, x^{*}, y\right)$ is a naïve (sophisticated) SC equilibrium. A naïve (sophisticated) SC equilibrium $\left(p^{*}, q^{*}, x^{*}, y^{*}\right)$ is said to be allocationally equivalent to a naïve (sophisticated) complete equilibrium if there are $p \in P, q \in Q$ and $y \in Y$ such that $\left(p, q, x^{*}, y\right)$ is a naïve (sophisticated) complete equilibrium.

In complete equilibria, relative prices are expected to remain unchanged. This is proven in the following theorem.

Theorem 5.3 Let the economy $\mathcal{E}$ be locally non-satiated and let $\left(p^{*}, q^{*}, x^{*}, y^{*}\right)$ be a naïve (sophisticated) complete equilibrium of $\mathcal{E}$. Then for every $s, s^{\prime}$ and every $t, \tau$ with $t<\tau$ there must be $\mu_{s^{\prime}(\tau) \mid s(t)} \in \mathbb{R}$ with

$$
q_{\left(s^{\prime}(\tau), T \mid s^{\prime}(\tau-1)\right) \mid s(t)}^{*}=\mu_{s^{\prime}(\tau) \mid s(t)}\left(p_{s^{\prime}(\tau) \mid s(t)}^{*}, q_{\left(\cdot \mid s^{\prime}(\tau)\right) \mid s(t)}^{*}\right) .
$$


In general, when preferences are allowed to be time-inconsistent, there is no link between naïve (sophisticated) complete equilibria and $\mathrm{AD}$ equilibria. In AD equilibria, because of the commitment power provided by the AD market structure, future selves of households have no influence. In naïve complete equilibria, future selves will typically deviate from the plans of current selves. In sophisticated complete equilibria, current selves are constrained by the optimizing behavior of future selves.

When preferences are time-consistent, every AD equilibrium is allocationally equivalent to some naïve (sophisticated) complete equilibrium. This is shown in the following two theorems.

Theorem 5.4 Let preferences in the economy $\mathcal{E}$ be time-consistent and let $\left(p^{*}, x^{*}\right)$ be an $A D$ equilibrium of $\mathcal{E}$. Then $\left(p^{*}, q^{*}, x^{*}, y^{*}\right)$ is a naïve complete equilibrium of $\mathcal{E}$, where $y_{\left(s^{\prime}(\tilde{\tau}) \mid s^{\prime}(\tau)\right) \mid s(t)}^{* h}=x_{s^{\prime}(\tilde{\tau}) \mid s(t)}^{* h}$ for every $h, s^{\prime}, s, \tilde{\tau}>\tau \geq t$ and $q_{\left(s^{\prime}(\tilde{\tau}) \mid s^{\prime}(\tau)\right) \mid s(t)}^{*}=$ $p_{s^{\prime}(\tilde{\tau}) \mid s(t)}^{*}$ for every $s^{\prime}, s, \tilde{\tau}>\tau \geq t$.

Theorem 5.5 Let preferences in the economy $\mathcal{E}$ be time-consistent and let $\left(p^{*}, x^{*}\right)$ be an $A D$ equilibrium of $\mathcal{E}$. Then $\left(p^{*}, q^{*}, x^{*}, y^{*}\right)$ is a sophisticated complete equilibrium of $\mathcal{E}$, where $y_{\left(s^{\prime}(\tilde{\tau}) \mid s^{\prime}(\tau)\right) \mid s(t)}^{* h}=x_{s^{\prime}(\tilde{\tau}) \mid s(t)}^{* h}$ for every $h, s^{\prime}, s, \tilde{\tau}>\tau \geq t$ and $q_{\left(s^{\prime}(\tilde{\tau}) \mid s^{\prime}(\tau)\right) \mid s(t)}^{*}=p_{s^{\prime}(\tilde{\tau}) \mid s(t)}^{*}$ for every $s^{\prime}, s, \tilde{\tau}>\tau \geq t$.

The following example shows that even when preferences are time-consistent, not every naïve complete equilibrium is allocationally equivalent to an $\mathrm{AD}$ equilibrium. As before, this result arises because naïve households form only rational price expectations.

Example 5.6 Consider Example 4.5. For two-period economies, the sequentially complete market structure is identical to the complete market structure. Thus, $\left(p^{*}, q^{*}\right.$, $\left.x^{*}, y^{*}\right)$ is also a naïve complete equilibrium. Again, there is no $p$ such that $(p,(2,2,1,1),(2,2,3,3))$ is an $\mathrm{AD}$ equilibrium.

Every sophisticated complete equilibrium, however, is allocationally equivalent to some $\mathrm{AD}$ equilibrium when preferences are time-consistent. Again, we need to make some additional assumptions.

Theorem 5.7 Let preferences be time-consistent, locally non-satiated, acyclic, and have open lower sections, and let consumption sets be closed and bounded from below. Let $\left(p^{*}, q^{*}, x^{*}, y^{*}\right)$ be a sophisticated complete equilibrium of $\mathcal{E}$ with $p^{*} \gg 0$. Then $\left(p, x^{*}\right)$ is an $A D$ equilibrium of $\mathcal{E}$, where $p_{1 \mid 1}=p_{1 \mid 1}^{*}$ and $p_{2, T \mid 1}=q_{2, T|1| 1}^{*}$.

So far we have studied the relation between AD equilibria on the one hand and SC and complete equilibria on the other hand. Now we will analyze the relation between the latter two. The next theorem shows that every naïve SC equilibrium is allocationally equivalent to some naïve complete equilibrium. Notice that there is no need to assume time-consistency to obtain this result.

Theorem 5.8 Let the economy $\mathcal{E}$ be locally non-satiated and let $\left(p^{*}, q^{*}, x^{*}, y^{*}\right)$ be a naïve $S C$ equilibrium of $\mathcal{E}$. Then there is $(p, q, y) \in P \times Q \times Y$ such that $\left(p, q, x^{*}, y\right)$ is a naïve complete equilibrium of $\mathcal{E}$. 
Surprisingly, there are naïve complete equilibria that are not allocationally equivalent to any naïve SC equilibrium, even when preferences are time-consistent. This is shown in the following example. The key insight is that the richer set of assets available under complete markets may lead to initial endowments in future periods that are not feasible under sequentially complete markets and that admit continuation equilibria that are not feasible in the sequentially complete markets setting.

Example 5.9 Consider an economy with three periods, no uncertainty, one good per period, and two households. Assume that each household initially owns one unit of the good in period 1 and two units of the good in period 2 and period 3, i.e., $e_{\cdot \mid 1}^{1}=e_{\cdot \mid 1}^{2}=(1,2,2)$.

Assume that both households have time-consistent preferences represented by the utility function

$$
u^{h}\left(x_{1 \mid 1}^{h}, x_{2 \mid 1}^{h}, x_{3 \mid 1}^{h}\right)=\sqrt{x_{1 \mid 1}^{h}}+\sqrt{x_{2 \mid 1}^{h}} \sqrt{x_{3 \mid 1}^{h}}+\sqrt{x_{3 \mid 1}^{h}} .
$$

Consider the complete market structure. Suppose that prices and price expectations in period 1 are given by $p_{\cdot \mid 1}^{*}=(1,1,1+\sqrt{2} / 2), q_{(\cdot \mid 1) \mid 1}^{*}=(1,1+\sqrt{2} / 2)$, and $q_{(\cdot \mid 2) \mid 1}^{*}=1+\sqrt{2} / 2$. Consider the case where price expectations are correct, so $p_{\cdot \mid 2}^{*}=(1,1+\sqrt{2} / 2), q_{(\cdot \mid 2) \mid 2}^{*}=1+\sqrt{2} / 2$, and $p_{\cdot \mid 3}^{*}=1+\sqrt{2} / 2$. It can easily be shown that with these prices both households expect to consume and actually will consume their initial endowments. Thus, these prices together with the consumption of the endowments and no trade in contingent commodities constitute a naïve complete equilibrium. It is easily verified that the same allocation can also be sustained by a naïve sequentially complete equilibrium.

In the complete market structure households have the option to trade contingent commodities in period 1 while still consuming their endowments in period 1 . Let prices and price expectations in period 1 be as before, $\bar{p}_{\cdot \mid 1}=(1,1,1+\sqrt{2} / 2)$, $\bar{q}_{(\cdot \mid 1) \mid 1}=(1,1+\sqrt{2} / 2)$, and $\bar{q}_{(\cdot \mid 2) \mid 1}=1+\sqrt{2} / 2$. Suppose that in period 1 households trade contingent commodities for period 2 and period 3 in such a way that

$$
\begin{aligned}
\bar{x}_{1 \mid 1}^{1} & =1, \\
\bar{y}_{(2 \mid 1) \mid 1}^{1} & =4+\sqrt{2}-\frac{2+\sqrt{2}}{2} \frac{8+8 \sqrt{2}}{4 \sqrt{2}-2 \sqrt{3}-2}, \\
\bar{y}_{(3 \mid 1) \mid 1}^{1} & =\frac{8+8 \sqrt{2}}{4 \sqrt{2}-2 \sqrt{3}-2} .
\end{aligned}
$$

Market clearing requires that $\bar{y}_{(2 \mid 1) \mid 1}^{2}=4-\bar{y}_{(2 \mid 1) \mid 1}^{1}$ and $\bar{y}_{(3 \mid 1) \mid 1}^{2}=4-\bar{y}_{(3 \mid 1) \mid 1}^{1}$. Notice that for $h=1,2, \bar{q}_{(2 \mid 1) \mid 1} y_{(2 \mid 1) \mid 1}^{h}+\bar{q}_{(3 \mid 1) \mid 1} y_{(3 \mid 1) \mid 1}^{h}=\bar{q}_{(2 \mid 1) \mid 1} e_{2 \mid 1}^{h}+\bar{q}_{(3 \mid 1) \mid 1} e_{3 \mid 1}^{h}$.

Households plan to retrade in period 2, for $h=1,2$,

$$
\bar{y}_{(3 \mid 2) \mid 1}^{h}=2 \text {, }
$$

so $\bar{x}_{\cdot \mid 1}^{h}=(1,2,2)$. 
Assume realized and expected prices in period 2 to be given by

$$
\begin{aligned}
\bar{p}_{\cdot \mid 2} & =\left(1, \frac{5+\sqrt{3}}{4}\right), \\
\bar{q}_{(3 \mid 2) \mid 2} & =\frac{5+\sqrt{3}}{4} .
\end{aligned}
$$

Then the optimal consumption for household 1 is $\bar{x}_{\cdot \mid 2}^{1}=(1,8 /(5+\sqrt{3}))$ and for household $2, \bar{x}_{\cdot \mid 2}^{2}=(3,4-8 /(5+\sqrt{3}))$. In period 3 it will follow that $\bar{x}_{3 \mid 3}^{1}=$ $\bar{y}_{(3 \mid 2) \mid 2}^{1}=8 /(5+\sqrt{3})$, and $\bar{x}_{3 \mid 3}^{2}=\bar{y}_{(3 \mid 2) \mid 2}^{2}=4-8 /(5+\sqrt{3})$. It can be verified that $(\bar{p}, \bar{q}, \bar{x}, \bar{y})$ is a naïve complete equilibrium.

In sequentially complete markets consuming the endowment in the first period implies no trade of contingent commodities. Therefore the allocation $\bar{x}$ cannot be achieved in a naïve sequentially complete equilibrium.

We have already mentioned that there is no relation between $\mathrm{AD}$ equilibria on the one hand and naïve (sophisticated) SC or naïve (sophisticated) complete equilibria on the other hand when preferences are not time-consistent. Theorem 5.8 shows that every naïve SC equilibrium is allocationally equivalent to some complete equilibrium and Example 5.9 shows that the converse is not true. When we consider sophisticated behavior, however, sophisticated SC equilibria are allocationally equivalent to sophisticated complete equilibria and vice versa. This is shown in the following theorem.

Theorem 5.10 Let the economy $\mathcal{E}$ be locally non-satiated. Every sophisticated SC equilibrium of $\mathcal{E}$ is allocationally equivalent to some sophisticated complete equilibrium of $\mathcal{E}$ and vice versa.

\section{Conclusion}

Arrow (1953) showed that every Pareto optimal allocation in an Arrow-Debreu economy can be achieved both by Arrow-Debreu markets and a particular sequentially complete market setting where first securities are traded and then spot markets are opened. Debreu (1959) claims that, when all contingent commodities can be traded in the first period and preferences of households do not change over time, there is no need for markets to reopen in later periods.

In this paper we allow preferences to be time-inconsistent and we analyze three market structures. In this richer environment, we reconsider the results of Arrow (1953) and Debreu (1959).

We first examine the Arrow-Debreu setting where all contingent commodities can be traded in the first period and where markets are not reopened in later periods. Next, we consider sequentially complete markets where goods on the spot markets and all one-period ahead contingent commodities can be traded in every state. Finally, we consider complete markets where all contingent commodities can be traded in every state. 
Following Pollak (1968) we make a distinction between naïve and sophisticated behavior. Naïve households do not realize that their preferences change over time, whereas sophisticated ones do. The latter will only make plans that they expect to carry out in the future.

For sophisticated households, we show that sequentially complete markets are allocationally equivalent to complete markets. For naïve households, every naïve sequentially complete equilibrium is allocationally equivalent to some naïve complete equilibrium, but the converse does not hold, even if we assume that preferences are time-consistent.

When preferences are time-consistent and households are naïve, every equilibrium in the Arrow-Debreu setting is allocationally equivalent to some naïve sequentially complete equilibrium and to some naïve complete equilibrium. When preferences are time-consistent and, in addition, households are sophisticated all three market market structures are allocationally equivalent.

Sophisticated behavior requires a lot from households, however. A more reasonable type of behavior would be "somewhat" sophisticated. Loewenstein et al. (2003) assume that households do make plans that they expect to stick to. However, they assume that households mispredict their future utility function and thus will want to reconsider their plans in the future. Here, we could model these households as having the same budget constraints as sophisticated households, but with different actual utility functions in later periods. For these households, it cannot be expected that they have perfect foresight of prices and thus, the results for the naïve households in this paper would apply. As soon as we allow at least one household not to be completely sophisticated, our paper shows that the degree of market completeness matters.

\section{Appendix}

Proof of Theorem 4.3 Let $\left(p^{*}, q^{*}, x^{*}, y^{*}\right)$ be a naïve SC equilibrium. Consider some $s, s^{\prime} \in S$ and $\tau, t \in T$ with $\tau>t$ and $s^{\prime}(t)=s(t)$. Since the economy is locally nonsatiated, it is evident that $p_{s^{\prime}(\tau) \mid s(t)}^{*} \neq 0$. Let $l$ be a commodity such that $p_{s^{\prime}(\tau), l \mid s(t)}^{*} \neq 0$.

Suppose there is $\tilde{l} \in L$ such that there is no $\mu \in \mathbb{R}$ with both

$$
\begin{aligned}
& \mu p_{s^{\prime}(\tau), l \mid s(t)}^{*}=q_{s^{\prime}(\tau), l \mid s(t)}^{*} \text { and } \\
& \mu p_{s^{\prime}(\tau), \tilde{l} \mid s(t)}^{*}=q_{s^{\prime}(\tau), \tilde{l} \mid s(t)}^{*} .
\end{aligned}
$$

Then it follows that

$$
p_{s^{\prime}(\tau), l \mid s(t)}^{*} q_{s^{\prime}(\tau), \tilde{l} \mid s(t)}^{*} \neq p_{s^{\prime}(\tau), \tilde{l} \mid s(t)}^{*} q_{s^{\prime}(\tau), l \mid s(t)}^{*}
$$

Let $h$ be a household such that $\succeq^{h, s(t)}$ is locally non-satiated in $s^{\prime}(\tau-1)$.

Case 1 Assume that

$$
p_{s^{\prime}(\tau), l \mid s(t)}^{*} q_{s^{\prime}(\tau), \tilde{l} \mid s(t)}^{*}>p_{s^{\prime}(\tau), \tilde{l} \mid s(t)}^{*} q_{s^{\prime}(\tau), l \mid s(t)}^{*}
$$


Now consider the vector $\Delta y_{\cdot \mid s(t)}^{h}$ defined by

$$
\Delta y_{s^{\prime \prime}\left(t^{\prime}\right), l^{\prime} \mid s(t)}^{h}= \begin{cases}p_{s^{\prime}(\tau), \tilde{l} \mid s(t)}^{*} & \text { if } s^{\prime \prime}\left(t^{\prime}\right)=s^{\prime}(\tau) \text { and } l^{\prime}=l, \\ -p_{s^{\prime}(\tau), l \mid s(t)}^{*} & \text { if } s^{\prime \prime}\left(t^{\prime}\right)=s^{\prime}(\tau) \text { and } l^{\prime}=\tilde{l} \\ 0 & \text { otherwise. }\end{cases}
$$

Then

$$
p_{s^{\prime}(\tau) \mid s(t)}^{*} \Delta y_{s^{\prime}(\tau) \mid s(t)}^{h}=0,
$$

i.e., in state $s(t)$ household $h$ could plan to buy $\Delta y_{\cdot \mid s(t)}^{h}$ in addition to $y_{\cdot \mid s(t)}^{* h}$, without changing income in state $s^{\prime}(\tau)$. Moreover,

$$
q_{s^{\prime}(\tau) \mid s(t)}^{*} \Delta y_{s^{\prime}(\tau) \mid s(t)}^{h}=q_{s^{\prime}(\tau), l \mid s(t)}^{*} p_{s^{\prime}(\tau), \tilde{l} \mid s(t)}^{*}-q_{s^{\prime}(\tau), \tilde{l} \mid s(t)}^{*} p_{s^{\prime}(\tau), l \mid s(t)}^{*}<0 .
$$

Therefore, buying $\Delta y_{\cdot \mid s(t)}^{h}$ in addition to $y_{\cdot \mid s(t)}^{* h}$ decreases expected expenditures in state $s^{\prime}(\tau-1)$, while not changing income in any other state. rium.

By local non-satiation this contradicts $\left(p^{*}, q^{*}, x^{*}, y^{*}\right)$ being a naïve $\mathrm{SC}$ equilib-

Case 2 Assume that

$$
p_{s^{\prime}(\tau), l \mid s(t)}^{*} q_{s^{\prime}(\tau), \tilde{l} \mid s(t)}^{*}<p_{s^{\prime}(\tau), \tilde{l} \mid s(t)}^{*} q_{s^{\prime}(\tau), l \mid s(t)}^{*}
$$

This case follows by repeating the argument of Case 1, replacing $\Delta y_{\cdot \mid s(t)}^{h}$ by $-\Delta y_{{ }_{\mid S(t)}}^{h}$.

It follows that for any $\tilde{l} \in L, \mu=q_{s^{\prime}(\tau), l \mid s(t)}^{*} / p_{s^{\prime}(\tau), l \mid s(t)}^{*}$ satisfies $\mu p_{s^{\prime}(\tau), \tilde{l} \mid s(t)}^{*}=$ $q_{s^{\prime}(\tau), \tilde{l} \mid s(t)}^{*}$.

Proof of Theorem 4.6 Let $\left(p^{*}, x^{*}\right)$ be an AD equilibrium. We define $q_{\cdot \mid s(t)}^{*}=p_{t+1, T \mid s(t)}^{*}$ for every $s(t)$. By local non-satiation of the economy we know that for every $s, s^{\prime} \in S$, $t, \tau \in T$ with $\tau>t$ and $s^{\prime}(t)=s(t)$ there is an $l_{s^{\prime}(\tau) \mid s(t)}$ with $p_{s^{\prime}(\tau), l_{s^{\prime}(\tau) \mid s(t)}^{*} \mid s(t)} \neq 0$. For every $s(t)$, we define $y_{\cdot s(t)}^{* h}$ recursively as follows. Start by setting, for every $h \in H$ and every $s^{\prime} \in S$ with $s^{\prime}(t)=s(t)$,

$$
y_{s^{\prime}(T), l_{s^{\prime}(T) \mid s(t)}^{* h} \mid s(t)}^{* h} \frac{p_{s^{\prime}(T) \mid s(t)}^{*}\left(x_{s^{\prime}(T) \mid s(t)}^{* h}-e_{s^{\prime}(T) \mid s(t)}^{h}\right)}{p_{s^{\prime}(T), l_{s^{\prime}(T) \mid s(t)}^{*} \mid s(t)}^{*}}+e_{S^{\prime}(T), l_{s^{\prime}(T) \mid s(t)}^{h} \mid s(t)}^{h},
$$

and $y_{s^{\prime}(T), l \mid s(t)}^{* h}=e_{s^{\prime}(T), l \mid s(t)}^{h}$ for every $l \neq l_{s^{\prime}(T) \mid s(t)}$. Then, continue by setting, for every $h \in H, t<\tau<T, s^{\prime} \in S$ with $s^{\prime}(t)=s(t)$, 


$$
\begin{aligned}
y_{s^{\prime}(\tau), l_{s^{\prime}(\tau) \mid s(t)}^{* h}(t)}= & \frac{p_{s^{\prime}(\tau) \mid s(t)}^{*}\left(x_{s^{\prime}(\tau) \mid s(t)}^{* h}-e_{s^{\prime}(\tau) \mid s(t)}^{h}\right)}{p_{s^{\prime}(\tau), l_{s^{\prime}(\tau)|s(t)| s(t)}^{*}} \mid e_{s^{\prime}(\tau), l_{s^{\prime}(\tau)|s(t)| s(t)}^{h}}} \\
& +\frac{\sum_{s^{\prime}(\tau+1) \in s^{\prime+}(\tau)}\left(p_{s^{\prime}(\tau+1) \mid s(t)}^{*} y_{s^{\prime}(\tau+1) \mid s(t)}^{* h}-p_{s^{\prime}(\tau+1) \mid s(t)}^{*} e_{s^{\prime}(\tau+1) \mid s(t)}^{h}\right)}{p_{s^{\prime}(\tau), l_{s^{\prime}(\tau) \mid s(t)} \mid s(t)}^{h}}
\end{aligned}
$$

and $y_{s^{\prime}(\tau), l \mid s(t)}^{* h}=e_{s^{\prime}(\tau), l \mid s(t)}^{h}$ for every $l \neq l_{s^{\prime}(\tau) \mid s(t)}$.

Then it holds that

$$
p_{s^{\prime}(T) \mid s(t)}^{*} y_{S^{\prime}(T) \mid s(t)}^{* h}=p_{s^{\prime}(T) \mid s(t)}^{*} x_{s^{\prime}(T) \mid s(t)}^{* h}
$$

for all $s, s^{\prime}, t, h$, and

$$
\begin{aligned}
& p_{s^{\prime}(\tau) \mid s(t)}^{*} x_{s^{\prime}(\tau) \mid s(t)}^{* h}+\sum_{s^{\prime}(\tau+1) \in s^{\prime+}(\tau)} p_{s^{\prime}(\tau+1) \mid s(t)}^{*} y_{s^{\prime}(\tau+1) \mid s(t)}^{* h} \\
& =p_{s^{\prime}(\tau) \mid s(t)}^{*} y_{s^{\prime}(\tau) \mid s(t)}^{* h}+\sum_{s^{\prime}(\tau+1) \in s^{\prime}(\tau)} p_{s^{\prime}(\tau+1) \mid s(t)}^{*} e_{s^{\prime}(\tau+1) \mid s(t)}^{h} .
\end{aligned}
$$

for all $s, s^{\prime}, h, t \leq \tau<T$.

We claim that $\left(p^{*}, q^{*}, x^{*}, y^{*}\right)$ is a naïve sequentially complete equilibrium.

By summing the budget constraints over all households, using that

$$
\sum_{h \in H} x_{\cdot \mid s(t)}^{* h}=\sum_{h \in H} e_{\cdot \mid s(t)}^{h}
$$

we obtain, for every $s, t$,

$$
\sum_{h \in H} y_{\cdot \mid s(t)}^{* h}=\sum_{h \in H} e_{\cdot \mid s(t+1)}^{h}
$$

It therefore holds that conditions (b) and (c) of the definition of a naïve sequentially complete equilibrium are satisfied. It only remains to be checked that condition (a) is satisfied as well.

Consider any $t, s, h$, and any $\left(x_{\cdot \mid s(t)}^{h}, y_{\cdot \mid s(t)}^{h}\right) \in{ }^{n} \bar{\gamma}_{s(t)}^{h}\left(p_{\cdot \mid s(t)}^{*}, q_{\cdot \mid s(t)}^{*}, y_{s(t) \mid s(t-1)}^{* h}\right.$, $e_{t+1, T \mid s(t)}^{h}$. By summing up budget constraints, we have that

$$
\begin{aligned}
& p_{\cdot \mid s(t)}^{*} x_{\cdot \mid s(t)}^{h}+p_{t+1, T \mid s(t)}^{*} y_{t+1, T \mid s(t)}^{h} \\
& \quad \leq p_{s(t) \mid s(t)}^{*} y_{s(t) \mid s(t-1)}^{* h}+p_{t+1, T \mid s(t)}^{*} e_{t+1, T \mid s(t)}^{h}+p_{t+1, T \mid s(t)}^{*} y_{t+1, T \mid s(t)}^{h},
\end{aligned}
$$

so,

$$
p_{\cdot \mid s(t)}^{*} x_{\cdot \mid s(t)}^{h} \leq p_{s(t) \mid s(t)}^{*} y_{s(t) \mid s(t-1)}^{* h}+p_{t+1, T \mid s(t)}^{*} e_{t+1, T \mid s(t)}^{h} .
$$


In addition, we have

$$
\begin{aligned}
& p_{s(\tau) \mid s(\tau)}^{*} x_{s(\tau) \mid s(\tau)}^{* h}+\sum_{s(\tau+1) \in s^{+}(\tau)} p_{s(\tau+1) \mid s(\tau)}^{*} y_{s(\tau+1) \mid s(\tau)}^{* h} \\
& \leq p_{s(\tau) \mid s(\tau)}^{*} y_{s(\tau) \mid s(\tau-1)}^{* h}+\sum_{s(\tau+1) \in s^{+}(\tau)} p_{s(\tau+1) \mid s(\tau)}^{*} e_{S(\tau+1) \mid s(\tau)}^{h}
\end{aligned}
$$

for every $\tau<t$.

By summing all these and by keeping in mind that $x^{*}$ is a time-consistent allocation, we find that $x_{\cdot \mid 1}^{* h} 2 x_{\cdot \mid s(t)}^{h} \in \breve{\gamma}_{1}^{h}\left(p_{\cdot \mid 1}^{*}, e_{\cdot \mid 1}^{h}\right)$.

Now suppose that there are $t, s, h$ with

$$
\left(x_{\cdot \mid s(t)}^{* h}, y_{\cdot \mid s(t)}^{* h}\right) \notin^{n} \bar{\delta}_{s(t)}^{h}\left(p_{\cdot \mid s(t)}^{*}, q_{\cdot \mid s(t)}^{*}, y_{s(t) \mid s(t-1)}^{* h}, e_{t+1, T \mid s(t)}^{h}\right)
$$

Then there must be $\left(\hat{x}_{\cdot \mid s(t)}^{h}, \hat{y}_{\cdot \mid s(t)}^{h}\right) \in{ }^{n} \bar{\gamma}_{s(t)}^{h}\left(p_{\cdot \mid s(t)}^{*}, q_{\cdot \mid s(t)}^{*}, y_{s(t) \mid s(t-1)}^{* h}, e_{t+1, T \mid s(t)}^{h}\right)$ with $\hat{x}_{\cdot \mid s(t)}^{h} \succ^{h, s(t)} x_{\cdot \mid s(t)}^{* h}$. But then, by time-consistency of preferences, $x_{\cdot \mid 1}^{* h} 2 \hat{x}_{\cdot \mid s(t)}^{h} \succ^{h, 1}$ $x_{\cdot \mid 1}^{* h}$. By the discussion above we also have $x_{\cdot \mid 1}^{* h} 2 \hat{x}_{\cdot \mid s(t)}^{h} \in \breve{\gamma}_{1}^{h}\left(p_{\cdot \mid 1}^{*}, e_{\cdot \mid 1}^{h}\right)$. So $x_{\cdot \mid 1}^{* h} \notin$ $\breve{\delta}_{1}^{h}\left(p_{\cdot \mid 1}^{*}, e_{\cdot \mid 1}^{h}\right)$, a contradiction to the fact that $\left(p^{*}, x^{*}\right)$ is an $\mathrm{AD}$ equilibrium.

Proof of Theorem 4.7 Define $q^{*}$ and $y^{*}$ as in Theorem 4.6. We only need to verify whether condition (a) of the definition of a sophisticated SC equilibrium is satisfied. By the same argument as in Theorem 4.6 we know that for every $s$

$$
x_{\cdot \mid s(T)}^{* h} \in{ }^{s} \bar{\delta}_{s(T)}^{h}\left(p_{\cdot \mid s(T)}^{*}, y_{s(T) \mid s(T-1)}^{* h}\right)
$$

We continue the proof by an induction argument. Assume that, for some $t$,

$$
\left(x_{\cdot \mid s(\tau)}^{* h}, y_{\cdot \mid s(\tau)}^{* h}\right) \in{ }^{s} \bar{\delta}_{s(\tau)}^{h}\left(p_{\cdot \mid s(\tau)}^{*}, q_{\cdot \mid s(\tau)}^{*}, y_{s(\tau) \mid s(\tau-1)}^{* h}, e_{\tau+1, T \mid s(\tau)}^{h}\right)
$$

for every $\tau>t$, for every $s$. Suppose that

$$
\left(x_{\cdot \mid s(t)}^{* h}, y_{\cdot \mid s(t)}^{* h}\right) \notin s \bar{\delta}_{s(t)}^{h}\left(p_{\cdot \mid s(t)}^{*}, q_{\cdot \mid s(t)}^{*}, y_{s(t) \mid s(t-1)}^{* h}, e_{t+1, T \mid s(t)}^{h}\right)
$$

Then there must be $\left(\hat{x}_{\cdot \mid s(t)}^{h}, \hat{y}_{\cdot \mid s(t)}^{h}\right) \in{ }^{s} \bar{\gamma}_{s(t)}^{h}\left(p_{\cdot \mid s(t)}^{*}, q_{\cdot \mid s(t)}^{*}, y_{s(t) \mid s(t-1)}^{* h}, e_{t+1, T \mid s(t)}^{h}\right)$ with $\hat{x}_{\cdot \mid s(t)}^{h} \succ^{h, s(t)} x_{\cdot \mid s(t)}^{* h}$. The same argument as in the proof of Theorem 4.6 then leads to a contradiction.

Proof of Theorem 4.8 Let $\left(p^{*}, q^{*}, x^{*}, y^{*}\right)$ be a sophisticated SC equilibrium. By Theorem 4.4, for every $s, s^{\prime} \in S$ and every $t, \tau \in T$ with $\tau>t$ and $s^{\prime}(t)=s(t)$, there must be $\mu_{s^{\prime}(\tau) \mid s(t)}$ such that

$$
q_{s^{\prime}(\tau) \mid s(t)}^{*}=\mu_{s^{\prime}(\tau) \mid s(t)} p_{s^{\prime}(\tau) \mid s(t)}^{*}
$$


Suppose that $\mu_{s^{\prime}(\tau) \mid 1} \leq 0$ for some $s^{\prime}(\tau)$. Then $q_{s^{\prime}(\tau) \mid 1}^{*}=\mu_{s^{\prime}(\tau) \mid 1} p_{s^{\prime}(\tau) \mid 1}^{*} \leq 0$. Thus, it would be possible to increase available income in state $s^{\prime}(\tau)$ without increasing expenditures in state $s^{\prime}(\tau-1)$. A household $h$ that, from the perspective of period 1 , is locally non-satiated in state $s^{\prime}(\tau)$, could change consumption in state $s^{\prime}(\tau)$ and have a consumption plan that is strictly preferred to the current bundle. Since preferences are time-consistent, the new consumption bundle would also be consistent with sophisticated behavior. This yields a contradiction against $x^{*}$ being an equilibrium allocation. It follows that $\mu_{s^{\prime}(\tau) \mid 1}>0$ for every $s^{\prime}(\tau)$.

Let $p_{1 \mid 1}=p_{1 \mid 1}^{*}$ and $p_{s^{\prime}(\tau) \mid 1}=p_{s^{\prime}(\tau) \mid 1}^{*} \prod_{\tau^{\prime}=2}^{\tau} \mu_{s^{\prime}\left(\tau^{\prime}\right) \mid 1}$ for every $s^{\prime} \in S$ and every $\tau>1$. Also, let $p_{\cdot \mid s(t)}=p_{s(t), T \mid 1}$ for all $t \in T, s \in S$. It holds that $p \gg 0$ and therefore that $\breve{\gamma}_{1}^{h}\left(p_{\cdot \mid 1}, e_{\cdot \mid 1}^{h}\right)$ is compact.

Suppose that $\left(p, x^{*}\right)$ is not an $\mathrm{AD}$ equilibrium. Then there is $s(t)$ with $x_{\cdot \mid s(t)}^{* h} \notin$ $\breve{\delta}_{s(t)}^{h}\left(p_{\cdot \mid s(t)}, x_{s(t), T \mid s(t-1)}^{* h}\right)$. Since we have $x_{\cdot \mid s(t)}^{* h}=x_{s(t), T \mid s(t-1)}^{* h}$ for every $t>1$, it should hold that $s(t)=1$. We know that $x_{\cdot \mid 1}^{* h} \in \breve{\gamma}_{1}^{h}\left(p_{\cdot \mid 1}, e_{\cdot \mid 1}^{h}\right)$. So there is $\bar{x}_{\cdot \mid 1}^{h}$ with $\bar{x}_{\cdot \mid 1}^{h} \in \breve{\gamma}_{1}^{h}\left(p_{\cdot \mid 1}, e_{\cdot \mid 1}^{h}\right)$ and $\bar{x}_{\cdot \mid 1}^{h} \succ^{h, 1} x_{\cdot \mid 1}^{* h}$. Since $\succeq^{h, 1}$ is acyclic and has open lower sections and $\breve{\gamma}_{1}^{h}\left(p_{\cdot \mid 1}, e_{. \mid 1}^{h}\right)$ is compact, according to Bergstrom (1975) there exists a maximum on $\breve{\gamma}_{1}^{h}\left(p_{\cdot \mid 1}, e_{\cdot \mid 1}^{h}\right)$ with respect to $\succeq^{h, 1}$. Without loss of generality, $\bar{x}_{\cdot \mid 1}^{h}$ equals that maximum. We define $\bar{x}_{\cdot \mid s(t)}^{h}=\bar{x}_{s(t), T \mid 1}^{h}$ for every $s(t)$. By the same argument as in the proof of Theorem 4.6, there must be $\bar{y}$ with $\left(\bar{x}_{\cdot \mid 1}^{h}, \bar{y}_{\cdot \mid 1}^{h}\right) \in{ }^{n} \bar{\gamma}_{1}^{h}\left(p_{\cdot \mid 1}, p_{2, T \mid 1}, e_{\cdot \mid 1}^{h}\right)$ and $\bar{y}_{s(t+1), T \mid s(t)}^{h}=\bar{y}_{s(t+1), T \mid 1}^{h}$ for every $s(t)$. Moreover, from the opportunity sets, it follows that whenever there is $y_{1}^{h}$ with $\left(x_{1}^{h}, y_{1}^{h}\right) \in{ }^{n} \bar{\gamma}_{1}^{h}\left(p_{\cdot \mid 1}, p_{2, T \mid 1}, e_{\cdot \mid 1}^{h}\right)$, then there is $y_{1}^{\prime h}$ with $\left(x_{1}^{h}, y_{1}^{\prime h}\right) \in{ }^{n} \bar{\gamma}_{1}^{h}\left(p_{. \mid 1}^{*}, q_{. \mid 1}^{*}, e_{. \mid 1}^{h}\right)$ and vice versa.

Then, by definition of $\bar{x}_{\cdot \mid 1}^{h}$ and the fact that $\bar{x}_{\cdot \mid 1}^{h} \succ^{h, 1} x_{\cdot \mid 1}^{* h}$, there is $s(t)$ such that

$$
\left(\bar{x}_{\cdot \mid s(t)}^{h}, y_{\cdot \mid s(t)}^{h}\right) \notin{ }^{s} \bar{\delta}_{s(t)}^{h}\left(p_{\cdot \mid s(t)}^{*}, q_{\cdot \mid s(t)}^{*}, \bar{y}_{s(t) \mid s(t-1)}^{h}, e_{t+1, T \mid s(t)}^{h}\right)
$$

for any choice of $y_{\cdot \mid s(t)}^{h}$. Two cases can be distinguished.

Case 1 There exists $y_{\cdot \mid s(t)}^{h}$ with $\left(\bar{x}_{\cdot \mid s(t)}^{h}, y_{\cdot \mid s(t)}^{h}\right) \in{ }^{s} \bar{\gamma}_{s(t)}^{h}\left(p_{\cdot \mid s(t)}^{*}, q_{\cdot \mid s(t)}^{*}, \bar{y}_{s(t) \mid s(t-1)}^{h}\right.$, $\left.e_{t+1, T \mid s(t)}^{h}\right)$. Then there must be $\left(\hat{x}_{\cdot \mid s(t)}^{h}, \tilde{y}_{\cdot \mid s(t)}^{h}\right) \in{ }^{s} \bar{\gamma}_{s(t)}^{h}\left(p_{\cdot \mid s(t)}^{*}, q_{\cdot \mid s(t)}^{*}, \bar{y}_{s(t) \mid s(t-1)}^{h}\right.$, $\left.e_{t+1, T \mid s(t)}^{h}\right)$ with $\hat{x}_{\cdot \mid s(t)}^{h} \succ^{h, s(t)} \bar{x}_{\cdot \mid s(t)}^{h}$. By time-consistency of preferences, it follows that $\bar{x}_{\cdot \mid 1}^{h} 2 \hat{x}_{\cdot \mid s(t)}^{h} \succ^{h, 1} \bar{x}_{\cdot \mid 1}^{h}$. Also, it can be checked that $\bar{x}_{\cdot \mid 1}^{h} 2 \hat{x}_{\cdot \mid s(t)}^{h} \in \breve{\gamma}_{1}^{h}\left(p \cdot p_{\cdot \mid 1}, e_{\cdot \mid 1}^{h}\right)$. This contradicts the definition of $\bar{x}_{\cdot \mid 1}^{h}$. Thus, case 1 is not possible.

Case 2 There does not exist $y_{\cdot \mid s(t)}^{h}$ with $\left(\bar{x}_{\cdot \mid s(t)}^{h}, y_{\cdot \mid s(t)}^{h}\right) \in s \bar{\gamma}_{s(t)}^{h}\left(p_{\cdot \mid s(t)}^{*}, q_{\cdot \mid s(t)}^{*}\right.$, $\left.\bar{y}_{s(t) \mid s(t-1)}^{h}, e_{t+1, T \mid s(t)}^{h}\right)$. Since $\bar{x}_{\cdot \mid 1}^{h} \in \breve{\gamma}_{1}^{h}\left(p_{\cdot \mid 1}, e_{\cdot \mid 1}^{h}\right)$ and relative prices do not change over time and are correctly anticipated, this can only happen if there is $s\left(t^{\prime}\right)$ with $t^{\prime}>t$ and

$$
\left(\bar{x}_{\cdot \mid s\left(t^{\prime}\right)}^{h}, \tilde{y}_{\cdot \mid s\left(t^{\prime}\right)}^{h}\right) \notin s \bar{\delta}_{s\left(t^{\prime}\right)}^{h}\left(p_{\cdot \mid s\left(t^{\prime}\right)}^{*}, q_{\cdot \mid s\left(t^{\prime}\right)}^{*}, \bar{y}_{s\left(t^{\prime}\right) \mid s\left(t^{\prime}-1\right)}^{h}, e_{t^{\prime}+1, T \mid s\left(t^{\prime}\right)}^{h}\right)
$$

for every $\tilde{y}_{\cdot \mid s\left(t^{\prime}\right)}^{h}$. 
Next, as before, for $s\left(t^{\prime}\right)$ we can distinguish two cases. We repeat this reasoning until we end up at the last period. For the last period, we can no longer distinguish two cases. In the last period, only case 1 can happen. This again leads to a contradiction by a similar reasoning as before.

Proof of Theorem 5.3 Consider some $s, s^{\prime} \in S$ and $t, \tau \in T$ with $\tau>t$ and $s^{\prime}(t)=$ $s(t)$. Since the economy is locally non-satiated, it holds that $p_{s^{\prime}(\tau) \mid s(t)}^{*} \neq 0$. Let $l$ be a commodity such that $p_{s^{\prime}(\tau), l \mid s(t)}^{*} \neq 0$.

Apart from the fact that we have more cases, the remainder of the proof is analogous to the proof of Theorem 4.3. Suppose that there is no $\mu \in \mathbb{R}$ with both

$$
\begin{aligned}
& \mu p_{s^{\prime}(\tau), l \mid s(t)}^{*}=q_{\left(s^{\prime}(\tau), l \mid s^{\prime}(\tau-1)\right) \mid s(t)}^{*}, \\
& \mu p_{s^{\prime}(\tau), \tilde{l} \mid s(t)}^{*}=q_{\left(s^{\prime}(\tau), \tilde{l} \mid s^{\prime}(\tau-1)\right) \mid s(t)}^{*}
\end{aligned}
$$

or that there is no $\mu$ with both

$$
\begin{aligned}
\mu p_{s^{\prime}(\tau), l \mid s(t)}^{*} & =q_{\left(s^{\prime}(\tau), l \mid s^{\prime}(\tau-1)\right) \mid s(t)}^{*} \text { and } \\
\mu q_{\left(s^{\prime \prime}(\tilde{\tau}), \tilde{l} \mid s^{\prime}(\tau)\right) \mid s(t)}^{*} & =q_{\left(s^{\prime \prime}(\tilde{\tau}), \tilde{l} \mid s^{\prime}(\tau-1)\right) \mid s(t)}^{*} .
\end{aligned}
$$

Next a contradiction can be obtained and the proof can be finished in a similar way as in the proof of Theorem 4.3.

Proof of Theorem 5.4 Let prices be as described in the theorem. First of all, we need one observation. Consider

$$
\left(x_{\cdot \mid s(t)}^{h}, y_{\cdot \mid s(t)}^{h}\right) \in{ }^{n} \gamma_{s(t)}^{h}\left(p_{\cdot \mid s(t)}^{*}, q_{\cdot \mid s(t)}^{*}, y_{(\cdot \mid s(t-1)) \mid s(t-1)}^{* h}\right) .
$$

Then we have

$p_{s^{\prime}(\tau) \mid s(t)}^{*} x_{s^{\prime}(\tau) \mid s(t)}^{h}+p_{s^{\prime+}(\tau), T \mid s(t)}^{*} y_{\left(\tau+1, T \mid s^{\prime}(\tau)\right) \mid s(t)}^{h} \leq p_{s^{\prime}(\tau), T \mid s(t)}^{*} y_{\left(s^{\prime}(\tau), T \mid s^{\prime}(\tau-1)\right) \mid s(t)}^{h}$

for every $s^{\prime}, \tau \geq t$. By adding these over all $s^{\prime}(\tau)$ with $\tau \geq t$ and $s^{\prime}(t)=s(t)$, we get

$$
p_{\cdot \mid s(t)}^{*} x_{\cdot \mid s(t)}^{h} \leq p_{\cdot \mid s(t)}^{*} y_{(s(t), T \mid s(t-1)) \mid s(t-1)}^{h} .
$$

Thus, we also have

$$
p_{\cdot \mid s^{\prime}(t)}^{*} x_{\cdot \mid s^{\prime}(t)}^{* h} \leq p_{\cdot \mid s^{\prime}(t)}^{*} y_{\left(s^{\prime}(t-1), T \mid s^{\prime}(t-1)\right) \mid s^{\prime}(t)}^{* h}
$$

for every $s^{\prime}$. Similarly, for all $\tau<t$, we know that

$$
p_{s(\tau) \mid s(\tau)}^{*} x_{s(\tau) \mid s(\tau)}^{* h}+p_{\tau+1, T \mid s(\tau)}^{*} y_{(\tau+1, T \mid s(\tau)) \mid s(\tau)}^{* h} \leq p_{. \mid s(\tau)}^{*} y_{(s(\tau), T \mid s(\tau-1)) \mid s(\tau-1)}^{* h},
$$

by definition of $y^{*}$. Using that $p_{s(\tau), T \mid s(t)}^{*}=p_{\cdot \mid s(\tau)}^{*}$, and taking the sum of Eq. (2) for all $s^{\prime}(t) \neq s(t)$, Eqs. (1) and (3) for all $s(\tau)$ with $\tau<t$, we obtain $x_{\cdot \mid 1}^{* h} 2 x_{\cdot \mid s(t)}^{h} \in$ $\breve{\gamma}_{1}^{h}\left(p_{\cdot \mid 1}^{*}, e_{\cdot \mid 1}^{h}\right)$. 
With this observation in mind, we can continue the proof. Since $\left(p^{*}, x^{*}\right)$ is an $\mathrm{AD}$ equilibrium and by definition of $y^{*}$, it follows immediately that conditions (b) and (c) of the definition of a naïve complete equilibrium are satisfied. It remains to be shown that condition (a) is satisfied as well. Suppose there are $t, s(t), h$ with $\left(x_{\cdot \mid s(t)}^{* h}, y_{\cdot \mid s(t)}^{* h}\right) \notin^{n} \delta_{s(t)}^{h}\left(p_{\cdot \mid s(t)}^{*}, q_{\cdot \mid s(t)}^{*}, y_{(\cdot \mid s(t-1)) \mid s(t-1)}^{* h}\right)$. Then there must be

$$
\left(\hat{x}_{\cdot \mid s(t)}^{h}, y_{\cdot \mid s(t)}^{h}\right) \in{ }^{n} \gamma_{s(t)}^{h}\left(p_{\cdot \mid s(t)}^{*}, q_{\cdot \mid s(t)}^{*}, y_{(\cdot \mid s(t-1)) \mid s(t-1)}^{* h}\right)
$$

with $\hat{x}_{\cdot \mid s(t)}^{h} \succ^{h, s(t)} x_{\cdot \mid s(t)}^{* h}$. But then, by time-consistency of preferences, $x_{\cdot \mid 1}^{* h} 2 \hat{x}_{\cdot \mid s(t)}^{h} \succ^{h, 1}$ $x_{\cdot \mid 1}^{* h}$. By our observation, it follows that $x_{\cdot \mid 1}^{* h} 2 \hat{x}_{\cdot \mid s(t)}^{h} \in \breve{\gamma}_{1}^{h}\left(p_{\cdot \mid 1}^{*}, e_{\cdot \mid 1}^{h}\right)$. So $x_{\cdot \mid 1}^{* h} \notin$ $\breve{\delta}_{1}^{h}\left(p_{\cdot \mid 1}^{*}, e_{\cdot \mid 1}^{h}\right)$, contradicting that $\left(p^{*}, x^{*}\right)$ is an $\mathrm{AD}$ equilibrium.

Proof of Theorem 5.5 First, of all, by definition of $p^{*}, q^{*}, x^{*}$, and $y^{*}$, it follows immediately that conditions (b), (c), (d), (e), (f), and (g) of the definition of a sophisticated complete equilibrium are satisfied. It remains to be shown that condition (a) is satisfied as well.

By the same argument as used in the proof of Theorem 5.4, it holds that

$$
\left(x_{\cdot \mid s(T)}^{* h}, y_{\cdot \mid s(T)}^{* h}\right) \in{ }^{s} \delta_{s(T)}^{h}\left(p_{\cdot \mid s(T)}^{*}, y_{\cdot \mid s(T-1)}^{* h}\right) .
$$

We proceed with an induction argument. Let $s \in S, t \in T$. Assume that

$$
\left(x_{\cdot \mid s(t+1)}^{* h}, y_{\cdot \mid s(t+1)}^{* h}\right) \in{ }^{s} \delta_{s(t+1)}^{h}\left(p_{\cdot \mid s(t+1)}^{*}, q_{\cdot \mid s(t+1)}^{*}, y_{(\cdot \mid s(t)) \mid s(t)}^{* h}\right)
$$

for every $s(t+1) \in s^{+}(t)$. Suppose that $\left(x_{\cdot \mid s(t)}^{* h}, y_{\cdot \mid s(t)}^{* h}\right) \notin s \delta_{s(t)}^{h}\left(p_{\cdot \mid s(t)}^{*}, q_{\cdot \mid s(t)}^{*}\right.$, $\left.y_{(\cdot \mid s(t-1)) \mid s(t-1)}^{* h}\right)$. Then there must be $\left(\hat{x}_{\cdot \mid s(t)}^{h}, \hat{y}_{\cdot \mid s(t)}^{h}\right) \in{ }^{s} \gamma_{s(t)}^{h}\left(p_{\cdot \mid s(t)}^{*}, q_{\cdot \mid s(t)}^{*}\right.$, $y_{(\cdot \mid s(t-1)) \mid s(t-1)}^{* h}$ with $\hat{x}_{\cdot \mid s(t)}^{h} \succ^{h, s(t)} x_{\cdot \mid s(t)}^{* h}$. But then, by time-consistency of preferences $x_{\cdot \mid 1}^{* h}<\hat{x}_{\cdot \mid s(t)}^{h} \succ^{h, 1} x_{\cdot \mid 1}^{* h}$. By the same argument as in the proof of Theorem 5.4 we also have $x_{\cdot \mid 1}^{* h}<\hat{x}_{\cdot \mid s(t)}^{h} \in \breve{\gamma}_{1}^{h}\left(p_{\cdot \mid 1}^{*}, e_{\cdot \mid 1}^{h}\right)$. This contradicts $\left(p^{*}, x^{*}\right)$ being an AD equilibrium.

Proof of Theorem 5.7 This proof is analogous to the proof for the sophisticated SC equilibrium in Theorem 4.8 .

Proof of Theorem 5.8 Since $\left(p^{*}, q^{*}, x^{*}, y^{*}\right)$ is a naïve SC equilibrium, it holds that

$$
\left(x_{\cdot \mid s(t)}^{* h}, y_{\cdot \mid s(t)}^{* h}\right) \in{ }^{n} \bar{\delta}_{s(t)}^{h}\left(p_{\cdot \mid s(t)}^{*}, q_{\cdot \mid s(t)}^{*}, y_{s(t) \mid s(t-1)}^{* h}, e_{t+1, T \mid s(t)}^{h}\right)
$$

For $s(t), s^{\prime}(\tau)$ with $\tau>t$, we define $\mu_{s^{\prime}(\tau) \mid s(t)}$ as the unique real number satisfying

$$
q_{s^{\prime}(\tau) \mid s(t)}^{*}=\mu_{s^{\prime}(\tau) \mid s(t)} p_{s^{\prime}(\tau) \mid s(t)}^{*},
$$

see Theorem 4.3. 
Next, we define $p_{s(t) \mid s(t)}=p_{s(t) \mid s(t)}^{*}$,

$$
\begin{gathered}
p_{s^{\prime}(\tau) \mid s(t)}=p_{s^{\prime}(\tau) \mid s(t)}^{*} \prod_{\tau^{\prime}=t+1}^{\tau} \mu_{s^{\prime}\left(\tau^{\prime}\right) \mid s(t)}, \\
q_{\left(s^{\prime}(\tau), T \mid s^{\prime}(\tau-1)\right) \mid s(t)}=p_{s^{\prime}(\tau), T \mid s(t) .}
\end{gathered}
$$

Let $y_{\left(s^{\prime}(\tau+1) \mid s^{\prime}(\tau)\right) \mid s(t)}^{h}=y_{s^{\prime}(\tau+1) \mid s(t)}^{* h}$ and $y_{\left(s^{\prime}(\tau+2), T \mid s^{\prime}(\tau)\right) \mid s(t)}^{h}=e_{\cdot \mid s^{\prime}(\tau+2)}^{h}$ for every $\tau \geq t$ and every $s^{\prime}$ with $s^{\prime}(t)=s(t)$. Then

$$
\left(x_{\cdot \mid s(t)}^{* h}, y_{\cdot \mid s(t)}^{h}\right) \in{ }^{n} \gamma_{s(t)}^{h}\left(p_{\cdot \mid s(t)}, q_{\cdot \mid s(t)}, y_{s(t) \mid s(t-1)}^{* h}, e_{t+1, T \mid s(t)}^{h}\right) .
$$

Suppose that $\left(x_{\cdot \mid s(t)}^{* h}, y_{\cdot \mid s(t)}^{h}\right) \notin^{n} \delta_{s(t)}^{h}\left(p_{\cdot \mid s(t)}, q \cdot \mid s(t), y_{s(t) \mid s(t-1)}^{* h}, e_{t+1, T \mid s(t)}^{h}\right)$. Then there must be $\left(x_{\cdot \mid s(t)}^{h}, \tilde{y}_{\cdot \mid s(t)}^{h}\right) \in{ }^{n} \gamma_{s(t)}^{h}\left(p_{\cdot \mid s(t)}, q_{\cdot \mid s(t)}, y_{s(t) \mid s(t-1)}^{* h}, e_{t+1, T \mid s(t)}^{h}\right)$ with $x_{\cdot \mid s(t)}^{h} \succ^{h, s(t)} x_{\cdot \mid s(t)}^{* h}$. As in the proof of Theorem 4.6 it follows that there is $\widehat{y}_{\cdot \mid s(t)}^{h}$ with

$$
\left(x_{\cdot \mid s(t)}^{h}, \widehat{y}_{\cdot \mid s(t)}^{h}\right) \in{ }^{n} \bar{\gamma}_{s(t)}^{h}\left(p_{\cdot \mid s(t)}^{*}, q_{\cdot \mid s(t)}^{*}, y_{s(t) \mid s(t-1)}^{* h}, e_{t+1, T \mid s(t)}^{h}\right)
$$

This then contradicts the fact that $\left(x_{\cdot \mid s(t)}^{* h}, y_{\cdot \mid s(t)}^{* h}\right) \in{ }^{n} \bar{\delta}_{s(t)}^{h}\left(p_{\cdot \mid s(t)}^{*}, q_{\cdot \mid s(t)}^{*}, y_{s(t) \mid s(t-1)}^{* h}\right.$, $\left.e_{t+1, T \mid s(t)}^{h}\right)$.

Proof of Theorem 5.10 Step 1 Consider $p \in P, q \in Q, \bar{q} \in \bar{Q}$, and, for every $s, s^{\prime}$, for every $\tau, t$ with $\tau \geq t$ and $s^{\prime}(t)=s(t), \mu_{s^{\prime}(\tau) \mid s(t)} \in \mathbb{R}$ such that

$$
\begin{aligned}
p_{s(\tau), T \mid s(t)} & =p_{\cdot \mid s(\tau)}, \\
\bar{q}_{s^{\prime}(\tau) \mid s(t)} & =\mu_{s^{\prime}(\tau) \mid s(t)} p_{s^{\prime}(\tau) \mid s(t)} \\
q_{\left(s^{\prime}(\tau), T \mid s^{\prime}(\tau-1)\right) \mid s(t)} & =\mu_{s^{\prime}(\tau) \mid s(t)}\left(p_{s^{\prime}(\tau) \mid s(t)}, q_{\left(\cdot \mid s^{\prime}(\tau)\right) \mid s(t)}\right), \\
p_{s^{\prime}(\tau) \mid s(t)} & \neq 0 .
\end{aligned}
$$

For every $s(\tau)$, for every $y_{\cdot \mid s(\tau-1)}^{h} \in Y_{\cdot \mid s(\tau-1)}^{h}$, for every $\bar{y}_{\cdot \mid s(\tau-1)}^{h} \in \bar{Y}_{\cdot \mid s(\tau-1)}^{h}$, we will prove that whenever household $h$ starts with the same income in both market structures, i.e.,

$$
\begin{aligned}
& p_{s(\tau) \mid s(\tau)} y_{(s(\tau) \mid s(\tau-1)) \mid s(\tau-1)}^{h}+\sum_{s^{\prime}(\tau+1) \in s^{+}(\tau)} q_{\left(s^{\prime}(\tau+1), T \mid s(\tau)\right) \mid s(\tau)} y_{\left(s^{\prime}(\tau+1), T \mid s(\tau-1)\right) \mid s(\tau-1)}^{h} \\
& =p_{s(\tau) \mid s(\tau)} \bar{y}_{s(\tau) \mid s(\tau-1)}^{h}+q_{(\tau+1, T \mid s(\tau)) \mid s(\tau)} e_{\tau+1, T \mid s(\tau)}^{h},
\end{aligned}
$$

we have that:

$$
\left(x_{\cdot \mid s(\tau)}^{h}, y_{\cdot \mid s(\tau)}^{h}\right) \in{ }^{s} \gamma_{s(\tau)}^{h}\left(p \cdot|s(\tau), q \cdot| s(\tau), y_{(\cdot \mid s(\tau-1)) \mid s(\tau-1)}^{h}\right)
$$


implies that there is $\bar{y}_{\cdot \mid s(\tau)}^{h} \in \bar{Y}_{\cdot \mid s(\tau)}^{h}$ such that

$$
\left(x_{\cdot \mid s(\tau)}^{h}, \bar{y}_{\cdot \mid s(\tau)}^{h}\right) \in{ }^{s} \bar{\gamma}_{s(\tau)}^{h}\left(p_{\cdot \mid s(\tau)}, \bar{q}_{\cdot \mid s(\tau)}, \bar{y}_{s(\tau) \mid s(\tau-1)}^{h}, e_{\tau+1, T \mid s(\tau)}^{h}\right)
$$

and vice versa. We give a proof by induction.

[Step 1a] First of all, it is obvious that for every $\bar{y}_{S(T) \mid s(T-1)}^{h}, y_{s(T) \mid s(T-1)}^{h}$ with

$$
p_{\cdot \mid s(T)} \bar{y}_{s(T) \mid s(T-1)}^{h}=p \cdot \mid s(T) y_{s(T) \mid s(T-1)}^{h}
$$

it holds that

$$
s \bar{\gamma}_{s(T)}^{h}\left(p \cdot \mid s(T), \bar{y}_{s(T) \mid s(T-1)}^{h}\right)={ }^{s} \gamma_{s(T)}^{h}\left(p_{\cdot \mid s(T)}, y_{s(T) \mid s(T-1)}^{h}\right) .
$$

[Step 1b] Assume the result is true for every $s(\tau)$, where $\tau>t$.

Now assume that, in state $s(t)$, household $h$ starts with the same income in both market structures, i.e.

$$
\begin{aligned}
& p_{s(t) \mid s(t)} y_{(s(t) \mid s(t-1)) \mid s(t-1)}^{h}+\sum_{s^{\prime}(t+1) \in s^{+}(t)} q_{\left(s^{\prime}(t+1), T \mid s(t)\right) \mid s(t)} y_{\left(s^{\prime}(t+1), T \mid s(t-1)\right) \mid s(t-1)}^{h} \\
& =p_{s(t) \mid s(t)} \bar{y}_{s(t) \mid s(t-1)}^{h}+q_{(t+1, T \mid s(t)) \mid s(t)} e_{t+1, T \mid s(t)}^{h} .
\end{aligned}
$$

We first prove one direction of our result.

$(\Rightarrow)$ Let

$$
\left(x_{\cdot \mid s(t)}^{h}, y_{\cdot \mid s(t)}^{h}\right) \in{ }^{s} \gamma_{s(t)}^{h}\left(p \cdot|s(t), q \cdot| s(t), y_{(\cdot \mid s(t-1)) \mid s(t-1)}^{h}\right) .
$$

We define $\bar{y}_{\cdot \mid s(t)}^{h}$ recursively as follows. For every $s^{\prime}(\tau), s(t)$ there is a $l_{s^{\prime}}(\tau) \mid s(t)$ with

$$
p_{s^{\prime}(\tau), l_{s^{\prime}(\tau) \mid s(t)} \mid s(t)} \neq 0 \text {. }
$$

Start by setting, for every $h \in H$,

$$
\bar{y}_{s^{\prime}(T), l_{s^{\prime}(T) \mid s(t)} \mid s(t)}^{h}=\frac{p_{s^{\prime}(T) \mid s(t)}\left(x_{s^{\prime}(T) \mid s(t)}^{h}-e_{s^{\prime}(T) \mid s(t)}^{h}\right)}{p_{s^{\prime}(T), l_{s^{\prime}(T) \mid s(t)} \mid s(t)}}+e_{s^{\prime}(T), l_{s^{\prime}(T) \mid s(t)} \mid s(t)}^{h},
$$

and $\bar{y}_{s^{\prime}(T), l \mid s(t)}^{h}=e_{s^{\prime}(T), l \mid s(t)}^{h}$ for every $l \neq l_{s^{\prime}(T) \mid s(t)}$. Then, continue by setting, for every $h \in H, t<\tau<T, s^{\prime} \in S$ with $s^{\prime}(t)=s(t)$

$$
\begin{aligned}
\bar{y}_{s^{\prime}(\tau), l_{s^{\prime}(\tau) \mid s(t)} \mid s(t)}= & \frac{p_{s^{\prime}(\tau) \mid s(t)}\left(x_{s^{\prime}(\tau) \mid s(t)}^{h}-e_{s^{\prime}(\tau) \mid s(t)}^{h}\right)}{p_{s^{\prime}(\tau), l_{s^{\prime}(\tau)|s(t)| s(t)}}}+e_{s^{\prime}(\tau), l_{s^{\prime}(\tau) \mid s(t)} \mid s(t)} \\
& +\frac{\sum_{s^{\prime}(\tau+1) \in s^{\prime+}(\tau)}\left(\bar{q}_{s^{\prime}(\tau+1) \mid s(t)} \bar{y}_{s^{\prime}(\tau+1) \mid s(t)}^{h}-\bar{q}_{s^{\prime}(\tau+1) \mid s(t)} e_{s^{\prime}(\tau+1) \mid s(t)}^{h}\right)}{p_{s^{\prime}(\tau), l_{s^{\prime}(\tau) \mid s(t)} \mid s(t)}}
\end{aligned}
$$


and $y_{s^{\prime}(\tau), l \mid s(t)}^{* h}=e_{s^{\prime}(\tau), l \mid s(t)}^{h}$ for every $l \neq l_{s^{\prime}(\tau) \mid s(t)}$.

Then,

$$
\begin{aligned}
& p_{s(t+1) \mid s(t+1)} \bar{y}_{s(t+1) \mid s(t)}^{h}+\sum_{s^{\prime}(t+2) \in s^{+}(t+1)} q_{\left(s^{\prime}(t+2), T \mid s(t+1)\right) \mid s(t)} e_{s^{\prime}(t+2), T \mid s(t)}^{h} \\
& =p_{s(t+1) \mid s(t+1)} x_{s(t+1) \mid s(t)}^{h}+\sum_{s^{\prime}(t+2) \in s^{+}(t+1)} q_{\left(s^{\prime}(t+2), T \mid s(t+1)\right) \mid s(t)} x_{s^{\prime}(t+2), T \mid s(t)}^{h} .
\end{aligned}
$$

and

$$
\begin{array}{r}
p_{s(t+1) \mid s(t+1)} y_{(s(t+1) \mid s(t)) \mid s(t)}^{h}+\sum_{s^{\prime}(t+2) \in s^{+}(t+1)} q_{\left(s^{\prime}(t+2), T \mid s(t+1)\right) \mid s(t+1)} y_{\left(s^{\prime}(t+2), T \mid s(t)\right) \mid s(t)}^{h} \\
=p_{s(t+1) \mid s(t+1)} x_{s(t+1) \mid s(t)}^{h}+\sum_{s^{\prime}(t+2) \in s^{+}(t+1)} q_{\left(s^{\prime}(t+2), T \mid s(t+1)\right) \mid s(t+1)} x_{s^{\prime}(t+2), T \mid s(t)}^{h}
\end{array}
$$

so it follows that

$$
\begin{gathered}
p_{s(t+1) \mid s(t+1)} y_{(s(t+1) \mid s(t)) \mid s(t)}^{h}+\sum_{s^{\prime}(t+2) \in s^{+}(t+1)} q_{\left(s^{\prime}(t+2), T \mid s(t+1)\right) \mid s(t+1)} y_{\left(s^{\prime}(t+2), T \mid s(t)\right) \mid s(t)}^{h} \\
=p_{s(t+1) \mid s(t+1)} \bar{y}_{s(t+1) \mid s(t)}^{h}+\sum_{s^{\prime}(t+2) \in s^{+}(t+1)} q_{\left(s^{\prime}(t+2), T \mid s(t+1)\right) \mid s(t)} e_{s^{\prime}(t+2), T \mid s(t)}^{h} .
\end{gathered}
$$

Using our induction hypothesis, we obtain easily that

$$
\begin{aligned}
& \left(x_{s(t+1), T \mid s(t)}^{h}, \bar{y}_{s^{+}(t+1), T \mid s(t)}^{h}\right) \\
& \quad \in \bar{\delta}_{s(t+1)}^{h}\left(p_{s(t+1), T \mid s(t)}, \bar{q}_{s^{+}(t+1), T \mid s(t)}, \bar{y}_{s(t+1) \mid s(t)}^{h}, e_{t+2, T \mid s(t+1)}^{h}\right) .
\end{aligned}
$$

Since the income of household $h$ in state $s(t)$ is the same under both market structures, it follows that

$$
\left(x_{\cdot \mid s(t)}^{h}, \bar{y}_{\cdot \mid s(t)}^{h}\right) \in{ }^{s} \bar{\gamma}_{s(t)}^{h}\left(p_{\cdot \mid s(t)}, \bar{q}_{\cdot \mid s(t)}, \bar{y}_{s(t) \mid s(t-1)}^{h}, e_{t+1, T \mid s(t)}^{h}\right) .
$$

$(\Leftarrow) \quad$ The other direction of our result is straightforward, when following the approach used in Theorem 5.8.

Step 2 Let $\left(p^{*}, q^{*}, x^{*}, y^{*}\right)$ be a sophisticated SC equilibrium. By local non-satiation we know that for all $s^{\prime}(\tau), p_{s^{\prime}(\tau) \mid 1}^{*} \neq 0$. By Theorem 4.4 there is $\mu_{s^{\prime}(\tau) \mid 1} \in \mathbb{R}$ such that

$$
q_{s^{\prime}(\tau) \mid 1}^{*}=\mu_{s^{\prime}(\tau) \mid 1} p_{s^{\prime}(\tau) \mid 1}^{*}
$$

We define $q \in Q$ as follows.

For every $s^{\prime}(T)$,

$$
q_{\left(s^{\prime}(T) \mid s^{\prime}(T-1)\right) \mid 1}=\mu_{s^{\prime}(T) \mid 1}\left(p_{s^{\prime}(T) \mid 1}^{*}\right) .
$$


Next, recursively, for $\tau$ descending from $T-1$ to 2 , for every $s^{\prime}(\tau)$,

$$
q_{\left(s^{\prime}(\tau), T \mid s^{\prime}(\tau-1)\right) \mid 1}=\mu_{s^{\prime}(\tau) \mid 1}\left(p_{s^{\prime}(\tau) \mid 1}^{*}, q_{\left(\cdot \mid s^{\prime}(\tau)\right) \mid 1}\right) .
$$

Finally, for every $s, s^{\prime}$, for every $t, \tau$ with $\tau \geq t$ and $s^{\prime}(t)=s(t)$,

$$
q_{\left(\cdot \mid s^{\prime}(\tau)\right) \mid s(t)}=q_{\left(\cdot \mid s^{\prime}(\tau)\right) \mid 1} .
$$

From the previous analysis it follows that there is a $y$ such that $\left(p^{*}, q, x^{*}, y\right)$ is a sophisticated complete equilibrium.

Step 3 Let $\left(p^{*}, q^{*}, x^{*}, y^{*}\right)$ be a sophisticated complete equilibrium. Consider any $s, s^{\prime}$, any $t, \tau$ with $\tau \geq t$ and $s^{\prime}(t)=s(t)$. By local non-satiation we know that $p_{s^{\prime}(\tau) \mid s(t)}^{*} \neq 0$. We define

$$
\bar{q}_{s^{\prime}(\tau) \mid s(t)}=q_{\left(s^{\prime}(\tau) \mid s^{\prime}(\tau-1)\right) \mid s(t)}^{*} .
$$

From the previous analysis it follows that there is $y \in \bar{Y}$ such that $\left(p^{*}, \bar{q}, x^{*}, y\right)$ is a sophisticated SC equilibrium.

\section{References}

Arrow, K.J., Debreu, G.: Existence of an equilibrium for a competitive economy. Econometrica 22, 265290 (1954)

Arrow, K.J.: Le rôle des valeurs boursières pour la répartition la meilleure des risques. In: Econométrie, colloques internationaux du centre national de la recherce scientifique. 40, pp. 41-47 (1953); English version: The role of securities in the optimal allocation of risk-bearing. Rev Econ Stud 31, 91-96 (1964)

Bergstrom, T.C.: Maximal elements of acyclic relations on compact Sets. J Econ Theory 10, 403-404 (1975)

Chattopadhyay, S., Gottardi, P.: Stochastic OLG models, market structure, and optimality. J Econ Theory 89, 21-67 (1999)

Debreu, G.: Theory of Value. New York: Wiley (1959)

Donaldson, J.B., Selden, L.: Arrow-Debreu preferences and the reopening of contingent claims markets. Econ Lett 8, 209-216 (1981)

Drèze, J.H., Herings, P.J.J.: Sequentially complete markets remain incomplete. METEOR Research Memorandum 03/44, Maastricht University pp. 1-13 (2003)

Haller, H.: Non-market reopening, time-consistent plans and the structure of intertemporal preferences. Econ Lett 32, 1-5 (1990)

Hellwig, M.F.: A note on the implementation of rational expectations equilibria. Econ Lett 11, 1-8 (1983)

Herings, P.J.J., Rohde, K.I.M.: Time-inconsistent preferences in a general equilibrium model. Econ Theory $29,591-619$ (2006)

Loewenstein, G., O’Donoghue, T., Rabin, M.: Projection bias in predicting future utility. Q J Econ 118, $1209-1248$ (2003)

Luttmer, E.G.J., Mariotti, T.: Subjective discounting in an exchange economy. J Political Econ 111, 1-30 (2003)

Pollak, R.A.: Consistent planning. Rev Econ Stud 35, 201-208 (1968)

Radner, R.: Existence of equilibrium of plans, prices, and price expectations in a sequence of markets. Econometrica 40, 289-303 (1972)

Svensson, L.E.O.: Sequences of temporary equilibria, stationary point expectations and Pareto-efficiency. J Econ Theory 13, 169-183 (1976) 